\title{
Review
}

\section{Phase separation processes in polymer solutions in relation to membrane formation}

\author{
P. van de Witte ${ }^{1}$, P.J. Dijkstra, J.W.A. van den Berg, J. Feijen * \\ Department of Chemical Technology and Institute for Biomedical Technology, University of Twente, P.O. Box 217, 7500 AE Enschede The \\ Netherlands
}

Received 16 November 1995; revised 13 March 1996; accepted 14 March 1996

\begin{abstract}
This review covers new experimental and theoretical physical research related to the formation of polymeric membranes by phase separation of a polymer solution, and to the morphology of these membranes. Two main phase separation processes for polymeric membrane formation are discussed: thermally induced phase separation and immersion precipitation. Special attention is paid to phase transitions like liquid-liquid demixing, crystallization, gelation, and vitrification, and their relation to membrane morphology. In addition, the mass transfer processes involved in immersion precipitation, and their influence on membrane morphology are discussed.
\end{abstract}

Keywords: Membrane preparation and structure; Theory; Thermodynamics; Phase Transitions; Mass transfer models

\section{Introduction}

\subsection{Polymeric membranes}

Polymeric membranes have been developed for a variety of industrial applications [1-3]. Examples of industrial applications are microfiltration, ultrafiltration, reverse osmosis and gas separation. Each application imposes specific requirements on the membrane material and membrane structure. For microfiltration and ultrafiltration membranes, the porosity

\footnotetext{
${ }^{*}$ Corresponding author.

${ }^{1}$ Current address: Philips Research, Prof. Holstlaan 4, 5656 AA Eindhoven, The Netherlands.
}

and the pore sizes of the membrane determine the efficiency of filtration. For gas separation, the selectivity and the permeability of the membrane material determine the efficiency of the gas separation. Porosity is an important feature of membranes, even for gas separation membranes: thin nonporous and fragile active layers are frequently supported by a rigid, porous, sublayer.

There are several ways to prepare porous polymeric films, such as sintering, stretching, track etching and phase separation processes. The final morphology of the fibres and membranes obtained will vary greatly, depending on the properties of the materials and the process conditions. The majority of membranes are prepared by controlled phase separation of polymer solutions into two phases: one with a 
high polymer concentration, and one with a low polymer concentration. The concentrated phase solidifies shortly after phase separation, and forms the membrane. The performance of this membrane strongly depends on the morphology formed during phase separation, and subsequent (or almost simultaneous) solidification.

In this review, new insights (developed during the past 5-10 years) into the general physical principles of membrane formation by phase separation processes will be discussed. At present, there is no such discussion available in the literature, as much new work has been done in this field since the appearance of previous books and reviews [1-6]. Work regarding mass transfer processes during membrane formation, and phase separation mechanisms like crystallization and spinodal demixing will be emphasized.

The field is too large to enable us to present a detailed quantitative treatment of each of the physical processes involved in membrane formation. However, in our opinion a more general review on the physical aspects of membrane formation will be useful for groups and industries involved in the preparation of membranes. A detailed discussion of the advantages and disadvantages of the techniques, and the specific conditions for the preparation of specific membrane structures does not fall within the scope of this paper.

\subsection{Preparation of porous membranes by phase separation techniques}

Phase separation of polymer solutions can be induced in several ways. Before discussing these phase separation processes in detail, a short survey of the four main techniques for the preparation of polymeric membranes by controlled phase separation is presented [4-6].

- Thermally induced phase separation (TIPS). This method is based on the phenomenon that the solvent quality usually decreases when the temperature is decreased. After demixing is induced, the solvent is removed by extraction, evaporation or freeze drying.

- Air-casting of a polymer solution [7,8]. In this process, the polymer is dissolved in a mixture of a volatile solvent and a less volatile nonsolvent. During the evaporation of the solvent, the solubility of the polymer decreases, and then phase separation can take place.

- Precipitation from the vapour phase. During this process, phase separation of the polymer solution is induced by penetration of nonsolvent vapour in the solution.

- Immersion precipitation. A polymer solution is cast as a thin film on a support or extruded through a die, and is subsequently immersed in a nonsolvent bath. Precipitation can occur because the good solvent in the polymer solution is exchanged for nonsolvent.

The differences between the four techniques originate from differences in desolvation mechanisms.

Phase diagrams can predict, whether or not a solution of a certain polymer in a certain solvent is suitable for membrane formation. Binary phase diagrams - showing the phase boundaries as a function of temperature and composition - provide information for the TIPS process, whereas ternary isothermal phase diagrams are useful for the prediction of the phase transitions that could occur when phase separation is induced according to one of the other methods.

It should be kept in mind that such diagrams only predict which phase transitions a polymer solution can undergo during a membrane formation process. An equilibrium phase diagram provides a map for the different phase transitions that are favoured thermodynamically. The kinetics of phase separation processes determine whether or not the thermodynamically favoured transition will occur, and also to what extent the transition will take place. Nonequilibrium processes may play an important role during membrane formation.

In each of the next sections, attention will be paid to the thermodynamic possibilities and the kinetic aspects involved. Section 2 is devoted to the TIPS process. The TIPS process is conceptually more simple than the immersion precipitation process, and this facilitates the introduction of the basic thermodynamic and kinetic concepts. The immersion process will be discussed in section 3 . The available literature on the other two processes is limited. Hence, these processes will not be treated in detail. Nevertheless, many of the discussions concerning immersion precipitation and TIPS are also valuable for understanding air casting, and precipitation from 
the vapour phase. Immersion precipitation can be regarded as a combination of nonsolvent penetration and solvent elimination. However, the rate of mass exchange for air casting and nonsolvent penetration is much slower than for immersion precipitation.

\section{Thermally induced phase separation}

\subsection{Introduction}

Liquid-liquid demixing processes play an important role in most of the TIPS processes. In addition, crystallization of the polymer from solution, gelation (and vitrification) of the polymer solution, and associations between the components in solution can occur. Some of these processes can also induce the formation of structures in solution. The combinations of liquid-liquid demixing with crystallization of the polymer, vitrification, association, and also crystallization of the solvent are of special importance for the generation of porous structures. These transitions and combinations of transitions will be discussed in more detail in the next sections.

\subsection{Liquid-liquid demixing}

If a solution of a polymer in a solvent of lower molecular mass exhibits liquid-liquid demixing, then a strongly asymmetric liquid-liquid demixing gap is typical. A phase diagram for a binary polymersolvent system is represented schematically in Fig. 1 [9]. In this figure the temperature is plotted as a function of the polymer concentration of the polymer solution. At high temperatures the solution is still homogeneous. At lower temperatures liquid-liquid phase separation in a polymer poor and a polymer rich phase can take place. These systems are characterized by an upper critical solution temperature. It should be noted that, for entropic reasons, many polymer-solvent systems also phase separate at temperatures close to or at elevated pressures even higher than the atmospheric boiling point of the solvent. These systems are characterized by a lower critical solution temperature. Liquid-liquid phase separation induced by heating the polymer solution is seldom used to make porous structures.

The boundary of the liquid-liquid demixing gap

\section{P. van de Witte et al.}

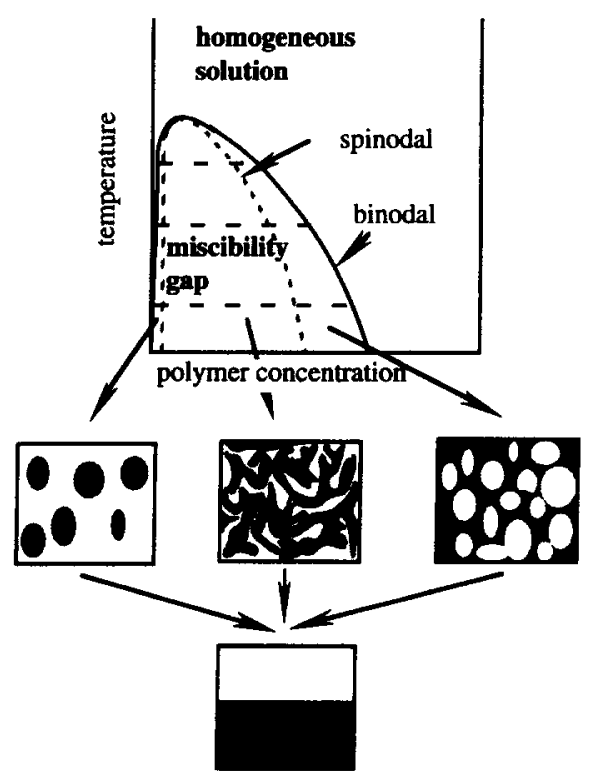

Fig. 1. A schematic representation of a binary phase diagram of a polymer solution showing a liquid--liquid demixing gap. In addition, the possible structure formation in the different parts of the liquid-liquid demixing gap is indicated: nucleation and growth of the polymer rich phase (left picture), bicontinuous morphology due to spinodal decomposition (middle picture) and nucleation and growth of a polymer poor phase (right picture).

is usually called the binodal, but for polydisperse polymers, the term "cloud point curve" is more appropriate. Usually the liquid-liquid demixing gap is subdivided into a region of spinodal demixing, bounded by the spinodal, and two regions of nucleation and growth between the binodal and the spinodal. Compositions that are in equilibrium are located on the binodal, and are connected by horizontal tie lines. It is important to note that the transition between binodal decomposition and spinodal decomposition should not be regarded as a sudden change in decomposition mechanism but as a gradual change [10].

The ratio of the equilibrium phases formed after demixing of a polymer solution with a specific composition is described by the lever rule. The point where the binodal and spinodal coincide is called the critical point. In the vicinity of the critical point, the 
compositions and amounts of the polymer rich phase and the polymer poor phase become more similar. For a monodisperse polymer, this point is located at the maximum of the binodal. When the polymer is polydisperse the picture becomes slightly more complicated. See [9] for a discussion of these effects.

Depending on the polymer concentration of the solution, liquid-liquid demixing proceeds according to different mechanisms. Compositions located between the binodal and the spinodal are metastable. This means that solutions are stable with respect to small fluctuations in the composition. However liquid-liquid demixing will occur when the fluctuations are large enough $[10,11]$. The degree of undercooling necessary to induce rapid binodal decomposition is very low for polymer solutions [12].

Liquid-liquid demixing takes place by nucleation and growth of droplets of a polymer poor phase when the original composition of the solution is located at polymer concentrations higher than that of the critical point. The solution demixes by nucleation and growth of droplets of a polymer rich phase when the polymer concentration is lower than that of the critical point (see Fig. 1). Once formed the droplet can grow because of the presence of a concentration gradient towards the droplet (Fig. 2) [10,11].

Solutions quenched into the area enclosed by the spinodal are unstable. All fluctuations in composition

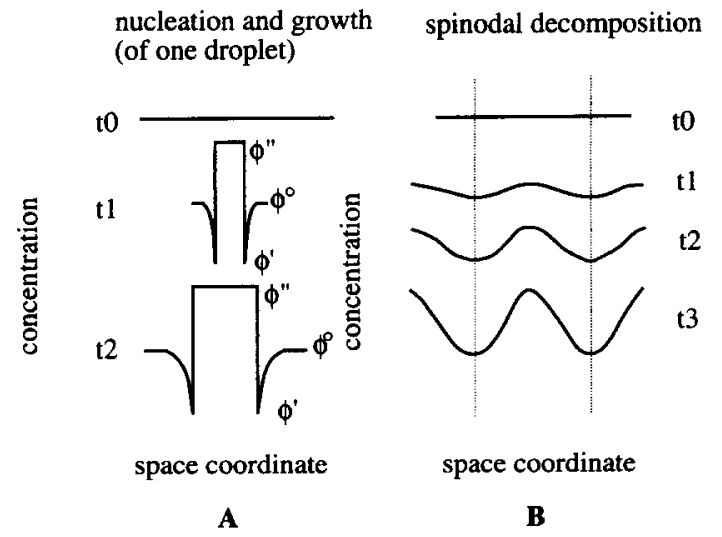

Fig. 2. (A) A schematic depiction of the nucleation and growth process (binodal demixing): $\phi^{0}$, original polymer concentration; $\phi^{\prime}$ and $\phi^{\prime \prime}$, binodal compositions. (B) A schematic picture of the concentration fluctuations as a function of time during spinodal decomposition. The vertical lines represent the wavelength of the fluctuation in the composition result in a decrease in free energy and trigger a wave of fluctuations through the solution [11]. Concentration fluctuations of different wave lengths and amplitude then develop. The amplitude of the fluctuations will increase because the molecules of both components will move from low concentration regions to high concentration regions. The wavelength with the highest growth rate will result in the most frequently found domain size.

When the fraction of the minor phase is sufficiently high, spinodal decomposition proceeds via the formation of bicontinuous structures. Both the polymer rich phase and the polymer poor phase are completely interconnected. As shown in Fig. 1, it is clear that during cooling, the spinodal area can only be entered directly at the critical point. In all other cases, the metastable area must first be passed. High cooling rates can be used to prevent demixing in the metastable area $[13,14]$.

The structures shown in Fig. 1 will grow and coarsen in time and eventually two fully separated layers may be obtained [13-18]. The driving force behind most of the coarsening processes is the minimization of the interfacial free energy. Coalescence results in a decrease of the number of droplets. Another type of coarsening is Ostwald ripening: the growth of larger structures at the expense of smaller structures (the droplets are not necessarily smooth and round). Ostwald ripening originates from the droplet size dependence of the concentration gradient near these droplets. Only droplets larger than a certain critical size are able to grow further. It is to be expected that coarsening by coalescence is the predominant mechanism for demixing polymer solutions [157].

Recently these phenomena have been studied in more detail for nucleation and growth by Lloyd and co-workers [153]. The radius of the polymer poor droplets increases exponentially in time, and is proportional to time raised to a certain power (the so-called scaling exponent). In the same way, the number of droplets decreases exponentially in time. The scaling exponent depends on many variables but is approximately $0.3[153,157]$. The growth rate decreases with increasing polymer concentration, and, to a lesser extent, also with decreasing quench temperature. During cooling, phase separation can occur repeatedly in the polymer rich and polymer poor 
phases that were formed in an earlier stage. This secondary phase separation can play an important role in the interconnectivity of cellular structures and in the generation of polymer spheres in membranes.

Structures formed by spinodal decomposition can coarsen too, by growth of some of the polymer domains which result from the dissolution of others [17]. Hydrodynamic flow will drive the structure towards a minimal surface free energy per unit volume [154]. Important quantities in the coarsening process are the interfacial tension between the two phases, and the viscosity of the matrix phase $[13,14,157]$. A remarkable description of structure formation through spinodal decomposition of polymer solutions was provided by Caneba and Soong [17]. By using some simplifying assumptions, the heat transfer and the spinodal decomposition pHrocess were simultaneously modelled. Reasonable agreement was found between the theoretically predicted characteristic length, and the one experimentally determined in the membrane.

Coarsening phenomena are the major barriers to being able to attribute obtained membrane structures to demixing processes. For instance, spinodally decomposed solutions gradually evolve into closed cell structures over time [154]. This shows that coarsening processes are very important, and determine the final size and interconnectivity of the porous structures of the membrane.

\subsection{Crystallization of the polymer}

Some polymers consist of molecules with sufficient regularity in the chain, so that these polymers can crystallize. Depending on the initial polymer concentration, the crystallization of such polymers from solution can lead to different morphologies: loose precipitates, or various types of "percolating structures", i.e. interconnected networks of crystallites (see also Fig. 3, to be discussed in more detail below). In many cases, such networks are called "gels" [20-23], although they represent only one form of the several possible types of solid-like phases also called "gels" (this use of this term will be discussed in more detail in Section 2.4 below).

Many factors influence the crystallization of polymers from solution [24]. The polymer concentration of the solution is a particularly important parameter.

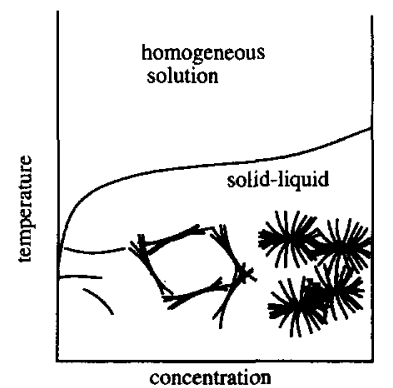

A

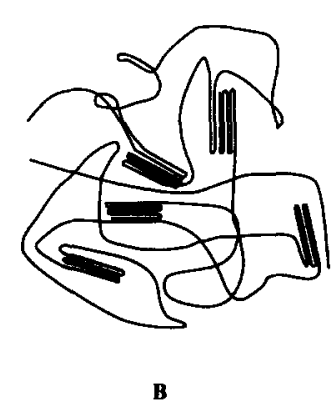

B
Fig. 3. Schematic equilibrium phase diagram for solid-liquid transition. (A) Morphologies of polymers obtained by crystallization (precipitation) from solution. Very low concentrations: single crystals, low concentrations: lamellar stacks, high concentrations: spherulites. (B) Molecular picture of a gel formed by crystallization from dilute solutions. For this picture it is assumed that the junctions are of a chain folded nature.

Crystallization of the polymer from dilute solutions usually results in the formation of chain folded lamellae [24,25]. The morphology of the lamellae that are obtained depends strongly on the characteristics of the polymer and on the conditions of crystallization. At higher polymer concentrations, suspensions of supramolecular architectures of these lamellae are obtained (see Fig. 3) [24-29]. Examples of supramolecular structures that are frequently isolated from gels are axialites and spherulites.

Kinetic aspects play a role in the supramolecular organization of the crystals. At sufficiently high concentrations, interpenetration and interlocking of these agglomerates of crystals can give rise to stiff brittle crystalline gels. Almost all crystalline polymers can form these kinds of gels. The formation of gels instead of unconnected precipitates can be promoted in several ways, e.g. (i) by stirring the solution or by introducing flow, (ii) through increasing the polymer concentration in the solution, or (iii) by increasing the molecular weight of the polymer $[22,23,30]$.

The size of the crystallites determines whether the gels are turbid or transparent [24]. Highly crystalline polymers will usually give relatively large crystallites, and turbid gels. Polymers with a low crystallinity (like random copolymers) can form transparent gels.

The fringed micelle model is sometimes postulated for very small crystallites [30,31]. The junc- 
tions between the polymer chains do not consist of chain folded crystallites, but instead the chains pass directly from one junction to another. The existence, however, of the fringed micellar crystallite is still matter of dispute [27,28]. For polyethylene-copolymers, with a low crystallinity, some properties of the crystallites in the gel (like heat and temperature of fusion) have been found to be exactly the same as the properties of the chain folded crystallites precipitated from dilute solutions. This led Mandelkern to the conclusion that the junctions in poorly crystalline copoly(ethylene-butene) gels were still chain folded crystallites, and not fringed micelle crystallites $[27,28]$. The extent of supramolecular organization of the lamellae decreased with decreasing crystallinity.

It should be noted that the ultimate morphology of the crystallites also depends on the kinetics of nucleation and growth of the crystallites. Due to the large activation energies necessary for the formation of crystalline nuclei and the limited growth rate of the nuclei, the crystallization curve is situated at much lower temperatures than the melting curve, obtained by reheating a crystallized solution. This melting curve (i.e. melting temperature as a function of polymer concentration) can be described at least qualitatively by an equilibrium theory, e.g. the Flory theory for the melting point depression of polymeric crystals in polymer solutions [32]. An example of such a curve is schematically presented in Fig. 3 . The trends in the morphology of the gels as a function of polymer concentration are also depicted.

It becomes more difficult to describe when polymers can crystallize into different crystalline modifications. Because the stability and the nucleation and growth rates of the different crystals can be different, gelation behaviour is very complex in such a case. Examples are syndiotactic polystyrene in decalin [33,34], poly-4-methyl-pentene in cyclohexane [35], polyvinylidene fluoride in $\gamma$-butyrolactone [36]. A special class of these crystallizing systems consist of mixtures of stereoisomeric polymers that can crystallize in stereocomplexes. Mixtures of stereoisomers can sometimes cocrystallize into special crystal conformations, which usually form gels. Important examples are poly(methyl methacrylates) and polylactides $[37,38]$. For some polymer-solvent combinations, the solvent can participate in the crystal structure $[19,39]$.

\subsection{Gelation and vitrification}

The solidification of polymer solutions is frequently denoted by the general term "gelation". The definition of "gelation" is not always clear, however, as it is also applied to cases where not "gels", but rather "glasses", are formed. In addition, the term "gel" itself is an ill-defined one. A (physical) "gel" can be defined as a network of physically crosslinked polymer chains with solvent trapped in the network. The structure of such a gel can have a transient character, because the physical crosslinks can reorganize under an applied stress. Physical gels can show an elastic response on fast deformation and flow behaviour in slow experiments (fast and slow with respect to the relaxation times of the polymer network).

Various types of intermolecular interactions can, in principle, give rise to an interconnected polymer network in solution. Microcrystallites, ionic interactions, hydrogen bonding, dipolar interactions, hydrophobic interactions, and solvent bridging may induce the formation of gels [19-21]. If the number of interactions is sufficiently high, and the lifetime of the interactions is sufficiently long, and if the interactions are sufficiently strong, the polymer solution can be considered as a "gel". Finally, it should be kept in mind that crystallization of polymers from solution is frequently regarded as a gel transition (see Section 2.3), and that on the other hand some groups even regard a solution with a viscosity of $10^{6}$ cP as a gel $[7,8]$. (thus, sometimes the term "viscosity transition" is applied instead of "gelation").

As suggested above, the formation of a glass from a polymer solution, is often regarded as a gel transition, though so research worker prefer the term "vitrification"' in such a case [40-42]. Strictly speaking, the Young's modulus of glasses is much higher than the Young's modulus of gels, and glasses should not be confused with gels. The glass transition of a polymer is decreased by the presence of small molecules. For polymers with high glass transition temperatures, a considerable amount of solvent can be added before the glass transition of the swollen, plastified, polymer reaches the temperature region where membrane formation takes place. In such cases substances much looser than a glass, with a rubber-like elasticity, can be formed, frequently 
referred to as "gels". (Ongoing loss of solvent, however, can cause such "gels" to change into a "glass", and "gelation" can be followed by "vitrification'.)

Thus, in our opinion it does not always make sense to treat gelation and vitrification as separate solidification processes in phase diagrams. We will use two terms, however, and the corresponding ones "gel" and "glass"' (and "glass transition"), and do so whenever possible in accordance with the literature cited. Only when sufficient viscoelastic data are available, as a function of measurement frequency, temperature, and solution composition, distinction between vitrification and gelation, and between the various types of gelation, really makes sense.

A large number of polymers can form thermoreversible gels in solvents: on cooling a polymer solution changes into a gel, at a certain temperature. On heating such a gel, it changes into the original solution, at the same temperature. The mechanisms involved in the gelation of polymer solutions have been the subject of extensive investigations during the last few years. The initial aim of the researchers was to provide a unifying scheme for the gelation of polymer solutions. However, it was soon recognized that the formation of gels can be induced in several ways. For extensive reviews of this subject, see [19] and [20]. In the following paragraphs, descriptions of gelation mechanisms will be discussed. Whenever possible examples will be provided. It has to be kept in mind that the mechanisms that are attributed to several gel forming systems are often only hypothetical mechanisms that have not been proven experimentally.

As previously mentioned in the introduction of this section, associations between components in solution due to specific interactions can result in the formation of gels. The number of articles that discuss the relevance of specific interactions for gelation is small. Nevertheless, it can be expected that these associations precede other phase transitions like crystallization, and occur more frequently than reported [43]. Theoretical discussions on gelation due to associations have been published by Coniglio, Joanny, Tanaka and Ferry [44-47]. Association between polymer segments usually can be expected to be more prominent at high polymer concentrations and under poor solvent conditions (in the vicinity of the

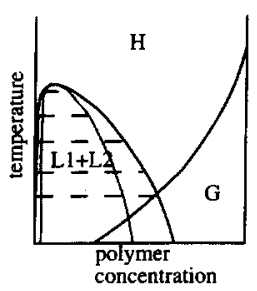

a)

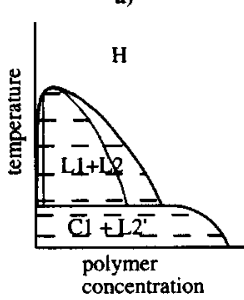

c) b1)

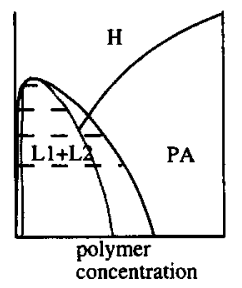

d)

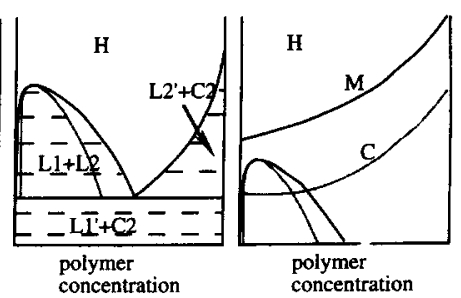

Fig. 4. Phase diagrams for combinations of phase transitions: (a) combination of liquid-liquid demixing and glass transition; (bl) equilibrium phase diagram for a combination of liquid-liquid demixing and crystallization of the polymer; (b2) nonequilibrium phase diagram for a combination of liquid-liquid demixing and crystallization of the polymer; (c) combination of liquid-liquid demixing and crystallization of the solvent; (d) combination of liquid-liquid demixing and polymer association. Symbols: $\mathbf{H}$, homogeneous solution; L1, dilute polymer solution; L2, concentrated polymer solution; G, glassy state; $\mathrm{C} 2$, polymer crystallites; PA, polymer association; $\mathrm{M}$, curve describing equilibrium melting point depression; $\mathrm{C}$, crystallization curve.

liquid-liquid demixing gap, see Fig. 4d) [48]. It has been noted by many authors that figures like Fig. $4 \mathrm{~d}$ show some resemblance to the theoretical phase diagrams of Daoud and Jannink showing the different concentration regimes in polymer solutions as a function of temperature $[47,48]$.

Stereoregular sequences may play a role in the gelation [19,31,49,50]. A particularly elucidating study was published by Mandelkern and co-workers. They studied the gelation of atactic, isotactic and epimerized polystyrene in cyclohexanol [29]. It was observed that the gelation mechanism outside of the liquid-liquid demixing gap changed gradually from solid-liquid demixing mediated gelation to association induced gelation, with decreasing stereoregularity of the polymer. Tan et al. also proposed an association mechanism for the gelation of atactic polystyrene in $\mathrm{CS}_{2}$ outside the demixing gap [50]. Gelation by side group association is a mechanism which has been postulated by Wolf and co-workers 
for the gelation of poly(butyl methacrylate) in poor solvents [51]. For polyvinylchloride in $\gamma$-butyrolactone, gelatin in water, and polyvinylalcohol in water association of polymer segments by hydrogen bonding is the first step in the gelation [46,52,53]. For these polymers, this process is followed by crystallization.

A couple of references present experimental evidence for complex formation of the polymer with the solvent [19]. The solvent can participate in amorphous complexes and form bridges between the different polymer chains. Many examples which have been studied, are isotactic polystyrene and atactic polystyrene in some solvents, like cis- or trans-decalin $[19,33,54-56]$. The exact mechanisms of these gelation processes are still under investigation.

Conformational transitions of the polymer from a random coil to a more ordered conformation can precede the gelation. Examples of synthetic polymers that demonstrate this transition are syndiotactic polystyrene in decalin and syndiotactic poly(methyl methacrylate) in $o$-xylene $[33,56,57]$. For biopolymers, gelation is frequently preceded by a coil-helix transition (e.g. polysaccharides in water) $[19,20,58]$. Gelation occurs by intertwining of multiple helices, aggregation of helices, or by crystallization processes.

\subsection{Combinations of liquid-liquid demixing and crystallization or liquid-liquid demixing and gela- tion}

As was discussed in the previous section, combinations of transitions are common in phase separations of polymer solutions. In this section, only combinations of liquid-liquid demixing and other transitions will be discussed, because these combinations are most relevant for the preparation of membranes. Phase transitions encountered most often in combination with liquid-liquid demixing are vitrification or gelation, crystallization of the polymer, and freezing of the solvent. In most cases, these transitions are useful to fix the structure of the solution at some stage during liquid-liquid demixing. However, interesting structures can also be obtained when liquid-liquid demixing and the other phase transitions occur simultaneously. Corresponding theoretical phase diagrams are given in Fig. 4. In this section, some attention will be paid to the influence of system variables on the morphology of the membrane.

\subsubsection{Liquid-liquid demixing and vitrification}

The most elegant technique for arresting stuctures in solution formed during liquid-liquid demixing is to continue cooling until the polymer rich phase passes the glass transition (Fig. 4a). The intersection point of the curve representing the glass transition of the polymer in solution and the cloud point curve is sometimes called the Berghmans point, referring to its discoverer $[40,42]$. The attractiveness of this combination is that the structures, as indicated schematically in Fig. 1, are "frozen in" without the interference of another structure-inducing transition. Keller et al. (atactic polystyrene-cyclohexanol) [59]; Aubert et al. [60]; Berghmans et al. (atactic polystyrenetrans-decalin, polymethacrylates-alcohols) $[40,41,61]$ and Torkelsson et al. (poly(methyl methacrylate) in several solvents) $[13,14]$ have explored the possibilities of this combination.

The thermodynamic background was provided by Frank and Keller [62]. Porous membranes can be obtained from the solidified demixed solution by extraction with a nonsolvent, by evaporation of the solvent, or by freeze drying. Aggregates of polymer beads were obtained at low polymer concentrations. At intermediate concentrations, bicontinuous network structures were obtained, and high polymer concentrations yielded cellular membranes. The membrane morphologies in solution could be further modified through coarsening, by keeping the solution for longer times above the vitrification boundary in the liquid-liquid demixing gap. Mandelkern and coworkers showed (for the system polystyrene-cyclohexanol) that an extra transition due to associations had to be included in the phase diagram [29]. Therefore, the explanation given above cannot be generalized to all amorphous polymer-solvent combinations.

The size and interconnectivity of the final membranes can be controlled by controlling the polymer concentration and the cooling scheme. For most applications, high porosity of the membrane and interconnectivity of the pores is desired. The structures formed by spinodal decomposition generally show very high interconnectivity and porosity. The 
interconnectivity of cellular structures decreases with increasing polymer concentrations. It has been shown that high cooling rates and viscous solvents are effective in decreasing the pore sizes of membranes prepared with specific polymer concentrations. Such conditions can also be used to create asymmetric membrane structures. When a polymer solution is introduced in between a material with a high heat conductivity and a material with a low heat conductivity (e.g. air) the effective cooling rate varies over the membrane thickness $[14,15,17]$. This variation in cooling rate will result in membranes with pore size gradients.

\subsubsection{Liquid-liquid demixing and crystallization of the polymer}

Another way in which the structure, formed by liquid-liquid demixing, can be stabilized, is through polymer crystallization (Fig. 4b). The thermodynamic background of combining crystallization with liquid-liquid demixing has been described by Burghardt [63]. Kinetic phenomena are important: liquid-liquid demixing processes generally start, and proceed, rapidly in polymer solutions (even for a small degree of undercooling), while for solid-liquid demixing processes generally nucleation and growth take place slowly, depending on the cooling rate, and on the degree of undercooling. In the interpretation of phase diagrams, the curve that describes the melting point depression should not be confused with the crystallization curve.

Liquid-liquid demixing can precede solid-liquid demixing even when solid-liquid demixing is favoured thermodynamically [64] and therefore the distance between the liquid-liquid demixing gap and the solid-liquid demixing gap is very important. The structure will be determined by solid-liquid demixing, when the solid-liquid demixing gap is located at much higher temperatures than the liquid-liquid demixing gap.

The phase separation of the polymer solution can become very complex, when the liquid-liquid demixing gap and the crystallization curve are located in the same temperature range. Different transitions can occur depending on the polymer concentration. These possibilities will be discussed in relation to the equilibrium phase diagram. Several phase equilibria can be distinguished [63].
Liquid-liquid demixing is favoured with low polymer concentrations, and at relatively high temperatures. Solid-liquid demixing is usually the result of the combination of high polymer concentrations and relatively high temperatures. Thermodynamically, a very dilute polymer solution is in equilibrium with a crystalline polymer phase at low temperatures (below the monotectic transition). In practice, the phase separation processes in this region are controlled by nonequilibrium phase separation processes. Liquid-liquid demixing will first occur, when the solution is cooled through the liquid-liquid demixing gap to a temperature below the monotectic transition. The polymer rich phase will be able to crystallize, when the solution is cooled to temperatures lower than the temperature at the intersection point of the crystallization curve and the liquid-liquid demixing gap. The structure of the liquid-liquid demixed solution is then stabilized. However, the morphology can be influenced by the crystallization process.

These phenomena have been studied in detail by Lloyd and co-workers for solutions of rapidly crystallizing polymers like polypropylene, polyvinylidene fluoride, polychlorotrifluoroethylene, poly-4methyl-1-pentene [16,65-69]. A similar study was performed by Aubert for isotactic polystyrene in nitrobenzene. [70] Schaaf et al. [71], Aerts et al. [72] and Mandelkern et al. [34] studied the crystallization and liquid-liquid demixing of polyethylene in some solvents. Cho et al. studied the gelation of polyvinylidene fluoride in $\gamma$-butyrolactone [36]. It is possible to obtain a great variety of membrane structures, ranging from crystallized polymer beads to spherulites with cellular or bicontinuous pore structures.

For slow crystallizing polymers, the influence of the crystallization on the structure formed during liquid-liquid demixing was much smaller than for rapidly crystallizing polymers $[34,70]$. The kinetics of both demixing processes play an important role in these processes. The competition between liquidliquid demixing and solid-liquid demixing is influenced by many factors. The solubility curve can be influenced to a lesser extent than the liquid-liquid demixing gap. As becomes clear from Flory's theory, the depression of the melting temperature of the polymer by solvents is, in most cases, determined by 
the polymer concentration, and by the heat of fusion of the polymer crystals. The polymer-solvent interaction parameter can be used to study the position of the liquid-liquid demixing gap with respect to the solid-liquid demixing gap. Higher values for the polymer-solvent interaction parameter shift the liquid-liquid demixing gap to higher temperatures with respect to the solubility curve. Additional solvents and nonsolvents can be added to the polymer solution to manipulate the interaction parameter. The rate of crystallization can be manipulated by adding crystallization additives to the solution, and by controlling the polymer concentration and the degree of undercooling [65-69].

\subsubsection{Liquid-liquid demixing and solvent crystal- lization}

Freezing of the solvent is another effective means of stabilizing the structure in the demixed polymer solution (Fig. 4c). Aubert demonstrated the importance of the relative positions, in the phase diagram, of the solvent freezing depression curve, and of the liquid-liquid demixing gap [60]. Porous membranes are obtained, with structures typical for the geometry of the solvent crystallites, when the solvent is frozen prior to liquid-liquid demixing $[68,70]$. The polymer is then expelled to the grain boundaries of the solvent crystallite. The pores are usually large and highly elongated. When liquid-liquid demixing takes place prior to the freezing of the solvent, there is no strong influence on the morphology.

\subsubsection{Liquid-liquid demixing and association of polymer molecules}

The structures which result from liquid-liquid demixing can also be stabilized by association of polymer molecules, or polymer side chains (Fig. 4d, see earlier section). The number of studies that report on the isolation of gel structures is rather small. It was demonstrated by Mandelkern, that morphologies which result from liquid-liquid demixing can be stabilized by associations in a similar way as described above (Section 2.4; see also [29]) for the glass transition. The structure of the gel formed by association phenomena, without the interference of liquid-liquid demixing, was "sheetlike" [34].

\section{Immersion precipitation}

\subsection{Introduction}

Membrane formation by immersion precipitation has been studied much less intensively than thermally induced phase separation. Nevertheless, a good picture of the process is now available. A schematic representation of membrane formation by immersion precipitation is presented in Fig. 5. Immersion precipitation is more complicated than thermally induced phase separation, because at least three components are involved, and because complex diffusion and convection processes play an important role. In the following sections, the phase behaviour of ternary systems and the exchange processes will be discussed. Finally, implications of phase transitions and mass transfer for the morphologies of membranes will be discussed. Only ternary combinations of polymers, solvents and nonsolvents will be discussed.

From the earlier discussion on binary systems, it is clear that a large number of phase transitions and combinations of phase transitions can play a role. Introducing a third component will make the phase diagrams even more complex. Fortunately, the complexity is reduced by the fact that the immersion precipitation process can be regarded as an isothermal process. For solvents and nonsolvents with very high heats of mixing the validity of this assumption is questionable.

In principle, most of the transitions mentioned for thermally induced phase separation can also occur during immersion precipitation. Three types of phase transitions which have general relevance for membrane formation will be discussed. As opposed to binary systems, ternary systems are discussed in the

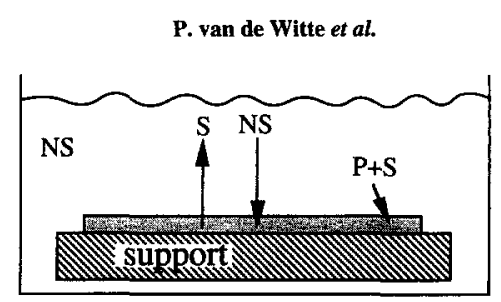

Fig. 5. Schematic depiction of the immersion precipitation process: $\mathrm{P}$, polymer; $\mathrm{S}$, solvent; NS, nonsolvent. 


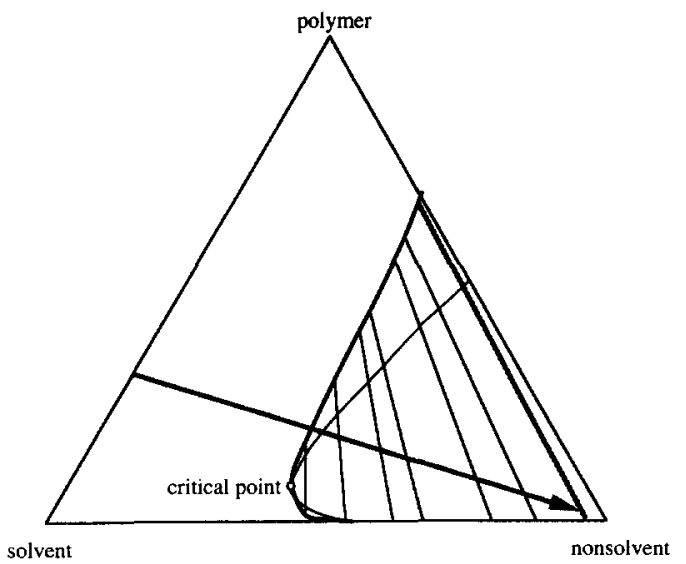

Fig. 6. Example of an isothermal phase diagram for mixtures of a polymer, a solvent and a nonsolvent. The arrow connects the initial composition of the casting solution to the final average composition of the entire system (nascent membrane + coagulation bath). The shaded tie line connects the final composition of the bath with the final composition of the polymer rich phase in the film.

literature almost exclusively in relation to generating porous morphologies. Liquid-liquid demixing plays a central role in this process. Therefore other phase transitions (vitrification) and solid-liquid demixing and gelation (crystallization of the polymer) will not be treated separately but in their relation with liquid-liquid demixing.

\subsection{Liquid-liquid demixing}

All of the possible combinations of three components can be plotted in a triangle. The corners represent the pure components, the axes the three binary combinations and a point in the triangle a ternary composition (Fig. 6). The phase diagram is divided into a homogeneous region, and an area representing a liquid-liquid demixing gap. The liquid-liquid demixing gap is entered when a sufficient amount of nonsolvent is present in the solution. In principle, the same three parts of the demixing gap are present as in the binary diagram. A metastable area exists between the spinodal and the binodal at low polymer concentrations, an unstable area is enclosed by the spinodal, and a second metastable area at higher polymer concentrations. The phase separation proceeds analogously with binary solutions.
A line is plotted in the phase diagram which connects the initial composition of the film to the final averaged composition of the film and the coagulation bath. The arrow does not represent the compositional change in the solution as a function of time. The components in the polymer solution will take a different composition path to the end condition. This topic will be dealt with in more detail in a later section of this paper (Section 3.3).

In the framework of the Flory-Huggins description of polymer solutions, the size and location of the demixing gap depends on the molar volumes of the components, the polymer-solvent interaction parameter, the polymer-nonsolvent interaction parameter, and the solvent-nonsolvent interaction parameter [73]. The influence of these variables on the resulting phase diagrams has been discussed in detail by Tompa, Altena and Tsay [73-75]. The effect of polydispersity of the polymer on these phase diagrams has been discussed by Koningsveld and Kamide [76,77]. The influence of the parameters can be summarized as follows.

- A polymer-nonsolvent interaction parameter $\left(\chi_{13}\right)$ determines, to a great extent, the surface area of the liquid-liquid demixing gap. High polymernonsolvent interaction parameters imply that the point of intersection of the demixing gap with the polymer-nonsolvent axis is located at very high polymer concentrations.

- Polymers and solvents with low mutual affinity (high $\chi_{23}$ ) increase the magnitude of the demixing gap, especially at low values of $\chi_{12}$.

- Low compatibility of solvent-nonsolvent mixtures (high $\chi_{12}$ ) results in large differences in solvent/nonsolvent ratio in the equilibrium phases.

- Solvents and nonsolvents with high mutual affinity (low $\chi_{12}$ ) strongly increase the magnitude of demixing gaps.

- In a first approximation (minor) changes in molecular weights, molecular weight distributions and molar volumes are negligible compared to the influence of interaction parameters.

The number of experimental ternary phase diagrams reported in the literature is limited. Some phase diagrams were determined by Strathmann for polyamides in a number of solvent / nonsolvent mixtures (Nomex) [4,5]. For polyurethane in DMF/water mixtures, the liquid-liquid demixing gap was deter- 
mined by Koenhen et al. [78] The phase behaviour of polysulfones and polyethersulfones in solvent/nonsolvent mixtures was studied by many groups [7985]. Ternary phase diagrams for cellulose acetate have also been frequently published [86-89]. The demixing gap for polyvinylidenedifluoride in some solvent/nonsolvent mixtures was reported by Bottino et al. [90] Utracki studied the system polystyrene-toluene-ethanol [91].

In general, the theoretical predictions for the influence of the parameters on the size and location of the liquid-liquid demixing gap are also found experimentally $[73,80,91]$. However, the quantitative correspondence between theoretical phase diagrams and experimental phase diagrams is rarely studied.

The determination of interaction parameters is usually rather time consuming. $\chi_{13}$ is difficult to determine, but can be calculated via swelling measurements [82]. $\chi_{12}$ can be calculated from activity data in the literature on solvent-nonsolvent mixtures [74] and $\chi_{23}$ can be obtained from light scattering or osmometry measurements $[9,32]$. In addition, vapour sorption can be used for the determination of polymer-solvent and polymer-nonsolvent interaction parameters. A more rapid indication for the (relative) values of some of these parameters can be obtained from intrinsic viscosity measurements $\left(\chi_{23}\right)$, and heats of mixing (usually available in literature, $\chi_{12}$ ). Even more rapid (although only qualitative) is the solubility parameter approach [92-94].

\subsection{Combinations of liquid-liquid demixing and other phase transitions}

All transitions,other than liquid-liquid demixing, are usually referred to as gel transitions in the literature on immersion precipitation. This includes viscosity "transitions" and transitions due to solidliquid demixing and vitrification.

Frequently the actual mechanism for gelation is not mentioned. For instance, in the case of cellulose acetate turbid and clear gels can be obtained. Certainly, crystallization plays a role in the gelation, but it is unclear whether this is a direct cause of the formation of the gel $[86,87]$. The formation of networks in solution due to hydrogen bonding was also suggested as a reason for the gelation [95].

\subsubsection{Liquid-liquid demixing and glass transition}

For amorphous polymers, membrane structures can be stabilized by vitrification. A phase diagram with a glass transition and a liquid-liquid demixing gap is presented in Fig. 7A. (compare with Fig. 4). Glass transition plotted as a function of composition

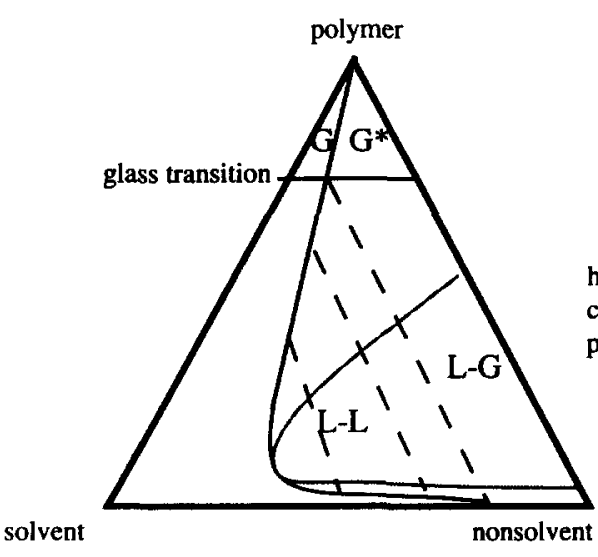

$\mathbf{A}$

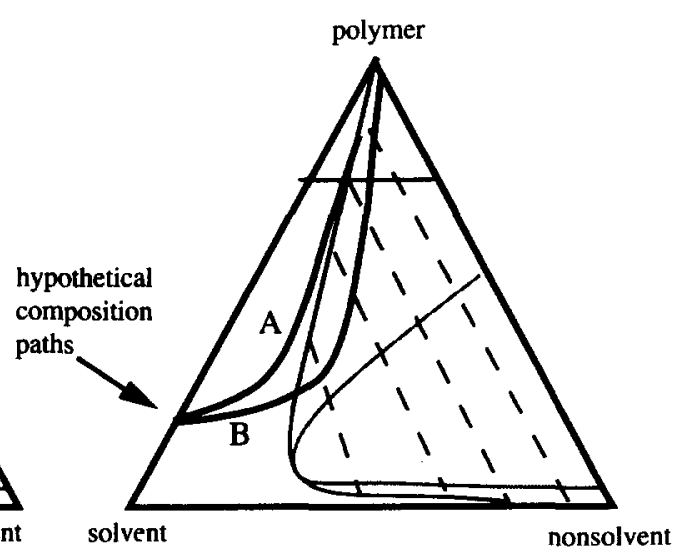

B

Fig. 7. Combination of a glass transition and a liquid-liquid demixing gap for a ternary system. (A) Phase diagram; (B) composition paths: L, liquid phase; $G$, glassy phase; $G^{*}$, metastable glassy state. For path $A$ the composition of the interface passes the vitrification boundary without demixing. For path B first demixing of the solution occurs. After demixing and further exchange the composition of the polymer rich phase passes the glass transition 
is frequently shown as a straight line. The location of this line is, among other parameters, dependent on the glass transition of the solvent and the nonsolvent. These values are usually in the same temperature range (ca. $100-150 \mathrm{~K}$ ), and the glass transition is therefore indicated by a horizontal line in the phase diagram. Several areas can be recognized in the phase diagram.

- The glassy state: the glassy state is divided into a homogenous stable glassy state and a metastable glassy state. In the latter case, the glass will phase separate by nucleation and growth. The rate of phase separation, however, will be slow.

- The demixing gap: in the part of the liquidliquid demixing gap which is not influenced by the glass transition, phase separation will proceed in the normal way.

The high polymer concentration end of tie lines located close to the polymer-nonsolvent axis lies in the glassy state. During the liquid-liquid demixing process, the polymer rich phase will vitrify. Callister et al. demonstrated that the phase separation process will continue to take place but at a much slower rate [42].

Using a mass transport model, Radovanovic demonstrated, that because of net outflow of, solvent the composition of the interface can reach a homogenous glassy state immediately after immersion (path $\mathrm{A}$ in Fig. 7B) $[96,97]$.

When the polymer concentration of the solution remains low, the composition will first enter the demixing gap (path B in Fig. 7B). During the demixing process, the exchange of solvent for nonsolvent continues, and the compositions of both the polymer rich phase and the polymer poor phase will move towards the polymer-nonsolvent axis. The composition of the polymer rich phase can therefore pass the glass transition.

For all amorphous polymers, the vitrification boundary can be expected to be a very important transition during membrane formation. For poly2,6-phenylene-1,4-oxide in trichloroethane and ethanol and for polysulfone in DMAc/water, the position of the glass transition in the phase diagram has been determined $[98,99]$.

Gaides demonstrated that the effect of the glass transition is already indicated with strongly increased viscosities at polymer concentrations far lower than

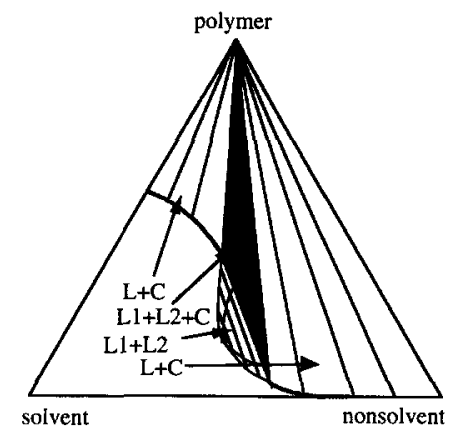

Fig. 8. Phase diagram of a three component system exhibiting both a solid-liquid and a liquid-liquid demixing gap. All equilibria are indicated by tie lines. The three phase equilibrium is shaded: L, liquid phase; $\mathrm{C}$, crystalline phase.

the polymer concentration at the glass transition [99]. Zeman arbitrarily defined the gel boundary for the system cellulose acetate-acetone-methylpentanediol with a high viscosity: $10^{6} \mathrm{cP}[7,8]$.

\subsubsection{Liquid-liquid demixing and solid-liquid demixing}

As already indicated in the section discussing binary systems, a large fraction of the polymers are able to exhibit other transitions besides vitrification and liquid-liquid demixing. Some of the polymers used for the preparation of membranes are semicrystalline. In this case, the same phenomena can be expected as for the binary systems.

The theoretical phase diagram becomes very complex, when crystallization of the polymer occurs concurrently with liquid-liquid demixing (Fig. 8). In some parts of the equilibrium phase diagram, equilibrium exists between a crystalline phase and a liquid phase, while in other parts of the phase diagram, equilibrium exists between two liquid phases.

With high polymer concentrations and low nonsolvent concentrations the polymer will crystallize from solution. At moderate polymer concentrations and with moderate nonsolvent concentrations liquid-liquid demixing will occur without the interference of solid-liquid demixing. At higher nonsolvent concentrations, liquid-liquid demixing will interfere with solid-liquid demixing. An important parameter is the distance between the solid-liquid demixing gap and the liquid-liquid demixing gap. It is possible that the solid-liquid demixing gap overlaps completely with the liquid-liquid demixing gap. 
Apart from thermodynamic properties, another important parameter is the rate of crystallization of the polymer. The fact that thermodynamically solid-liquid demixing is preferred over liquid-liquid demixing does not imply that kinetically solid-liquid demixing is favoured over liquid-liquid demixing (see TIPS section). This was demonstrated clearly in a DSC study for the system polylactidechloroform-methanol by Van de Witte et al. [147].

The possible influence of crystallization processes on phase diagrams was assessed already in earlier studies on membrane formation by Smolders and co-workers. The phase diagrams that were obtained resemble, to some extent, the theoretical diagrams. Phase diagrams were obtained in which the line separating the homogenous region from the inhomogeneous region was built up partly from the cloud point curve and partly from the gelation curve. Koenhen et al. measured the crystallization region and the cloud point curve in ternary solutions of polyurethanes in DMF/water mixtures [78]. DSC studies and turbity studies were performed by Smolders et al. on ternary solutions of cellulose acetate and PPO $[86,87,100,101]$.

Reuvers demonstrated the importance of the cooling rate on the phase separation of cellulose acetate in ternary solutions [86]. The gelation curve varied with the cooling rate, while the liquid-liquid demixing transition did not depend on the cooling rate. This study demonstrates that equilibrium transitions for gelation can only be obtained from the melting point data, and not from data obtained from cooling experiments. The location of the gelation boundary during the immersion precipitation process depends on the time scale in which the events take place.

Equilibrium melting transitions for cellulose acetate in ternary solutions were measured by Altena [87]. Further research on equilibrium phase behaviour of polyphenyleneoxide in trichlorethylene/ ethanol mixtures has been carried out by Burghardt et al. [98]. They presented a phase diagram similar to the one indicated in Fig. 8, and demonstrated that for this system crystallization is preferred thermodynamically over liquid-liquid demixing, spanning nearly the entire composition range.

Cheng and co-workers recently measured the solid-liquid demixing gaps for nylons in formic acid-water. An indication for the position of the liquid-liquid demixing gap can be obtained from the cloud point curves for the amorphous terpolymer of nylon-66/610/6 [102]. It was shown that, for these systems, the liquid-liquid demixing gap was located at higher nonsolvent concentrations than the solidliquid demixing gap. A similar conclusion was found by Bulte for nylon- 4,6 in the same solvent/nonsolvent mixture [103].

Experimental phase diagrams and theoretically calculated phase diagrams were compared by Van de Witte et al. for polylactide-solvent-nonsolvent systems [147]. The agreement of the calculated liquidliquid demixing gap with the experimental liquidliquid demixing gap was much better than the corresponding phase boundaries for solid-liquid demixing. Depending on the solvent-nonsolvent system and the composition of the solution the importance of solid-liquid demixing and liquid-liquid demixing could be shifted. Highly porous membranes, which are suitable for drug delivery, could be obtained for solid-liquid demixing induced fibre morphologies [147].

It should be kept in mind that some polymers which are usually referred to as amorphous, for example polysulfone and polyethersulfone, may be able to crystallize under some conditions. The oligomeric fraction of polysulfone can crystallize from solution [104]. Polyethersulfone can form crystalline complexes with some solvents (e.g. dichloromethane) [105].

\subsection{Mass transfer}

\subsubsection{Theoretical}

Many improvements have been made in the development of the extremely complex mass transfer models since the first model of Cohen et al. [96,97,106-112]. (For an introductory text on mass transfer see [113]). Three models that have been used frequently in literature are the model developed by Reuvers and co-workers [107,108], the model developed by Tsay and McHugh $[111,156]$ and the model developed by Cheng et al. [112].

These models start from basic diffusion equations and continuity equations for both the bath side and the film side. Using thermodynamics of irreversible processes, the fluxes of the three components were related to space derivatives of the chemical poten- 
tials of the three components. The resulting set of equations describes the composition of the film and the bath as a function of space coordinates and time. Input parameters for the equations are thermodynamic and kinetic parameters. It is very difficult to find a satisfactory thermodynamic description for the ternary system and suitable values for the kinetic input parameters at this stage of the model description (see remarks in [112]).

Crucial aspects of the mass transfer models are the boundary conditions and the initial conditions necessary to solve the differential equations. The most important assumptions are:

1. No convection occurs in the film side or the bath side, and diffusion is one-dimensional.

2. Instantaneous equilibrium exists at the interface between the bath side and the film side of the interface.

3. No polymer dissolves in the coagulation bath.

4. The model of Reuvers et al. assumes an infinite film thickness. This restriction is eliminated in the models of Tsay and Cheng.

5. Gradient energy terms can be neglected.

6. Demixing occurs by nucleation and growth of the polymer poor phase.

3.4.1.1. Referring to points $1+2$ above. These assumptions imply that homogeneous diffusion takes place in the polymer solution and in the bath. The chemical potentials of the components are assumed to be continuous in the entire system. Gaides and McHugh have demonstrated that the bath side of the process is, in fact, convection controlled [114]. Experimentally, the lack of homogeneous diffusion in the bath side can often be observed visually during the immersion precipitation process. In these cases, currents with a different refractive index can be identified.

The equilibrium assumption for the interface implies that immediately following immersion, the composition of the solution at the film side of the interface is connected by a tie line to the composition of the solution at the bath side of the interface. Yilmaz and McHugh argue that especially in the first moments after immersion of the polymer solution in the coagulation bath the equilibrium condition should be replaced by a mass transfer coefficient formalism [109]. In order to include the contribution of convec- tion, extra parameters have to be introduced into the theory, and these are difficult to quantify.

Cheng et al. have modified the mass transfer equations for the nonsolvent bath by introducing a velocity of the nonsolvent bath parallel to the film [112].

3.4.1.2. Referring to point 3. This assumption implies that the solubility of the polymer in the solvent/nonsolvent mixture at the bath side of the interface is negligible. When the molecular weight of the polymer is sufficiently high and the polymer concentration at the interface is also high the polymer concentration at the dilute end of the tie line will be negligible. However, for low molecular weights and/or broad molecular weight distributions, the validity of this assumption is questionable. It may also be possible that due to the diffusion cross terms, the polymer can be dragged into the coagulation bath (compare [115]).

3.4.1.3. Referring to point 4. For predicting the membrane morphology the composition profile of the solution at the onset of demixing is the most important. The release of the infinite film thickness assumption is therefore a valuable improvement. The model, however, becomes computationally involved.

3.4.1.4. Referring to point 5. If compositional differences exist in solution, the free energy of an element in solution is a function of the composition and the composition gradient. For small concentration gradients, the driving force for the flux of a component can be approximated by the chemical potential gradient. A gradient energy term has to be included for large concentration gradients. For example, both gradient terms and chemical potential terms are included in the Cahn description for the spinodal decomposition process [11]. For modelling membrane formation, this contribution has been neglected (see for some remarks $[96,97])$.

3.4.1.5. Referring to point 6 . In the early model of Cohen et al., demixing can occur by spinodal decomposition [106]. In the models of Reuvers and Tsay, it is assumed that demixing occurs immediately after the binodal is crossed. The spinodal region cannot be reached $[96,97,116,150]$. This is due to the fact that 
the thermodynamic driving force for diffusion becomes zero at the spinodal. Cheng et al. extended the calculations into the metastable area using the assumption that in the first moments after phase separation the composition of the polymer solution has not changed very much [112].

The influence of these assumptions on the conclusions drawn from the model calculations has never been addressed explicitly in the literature on mass transfer modelling. In spite of the drawbacks listed above, valuable predictions have been obtained from the diffusion models $[107,108,111]$. Especially the possibility to identify the parameters that are involved in mass transfer is an important result of mass transfer models.

\subsubsection{Predictions of the mass transfer models}

Mass transfer models describe the composition of the solution as a function of space coordinates and time. Composition paths can be derived from the model calculations. The composition path can be defined in two ways. The composition path can represent the composition range in the polymer solution between the support and the interface at a certain time. The composition path can also be defined as the composition of a certain well defined element in the solution as a function of time. As long as the infinite film thickness assumption is valid (which is true for the first time periods after immersion before precipitation), the composition path represents both the composition of an element in solution as a function of time and the composition range between interface and support. In the model of Reuvers et al. infinite film thickness is assumed [107]. In this case both definitions can be represented by a single composition path.

Reuvers et al. were the first to demonstrate theoretically that the mass transfer processes associated with most membrane forming systems can be divided in two categories: delayed demixing and instantaneous demixing [107,108]. In Fig. 9 the composition paths for both categories are presented schematically in a ternary phase diagram. During the delayed demixing process (path $I$ in Fig. 9) the composition of the entire solution remains in the homogeneous region of the phase diagram for a certain time period. This time period is called the delay time. During the delay time, the compositions

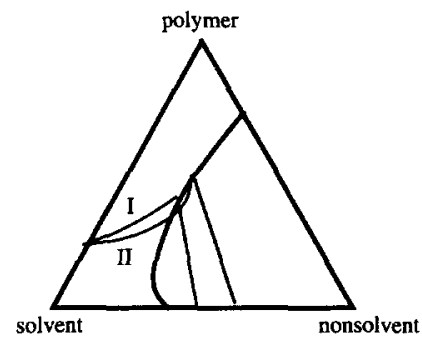

Fig. 9. Schematic depiction of the two different categories of composition paths in membrane formation. The thick line is the binodal and the thin straight lines represent tie lines. I, Delayed demixing. II, Instantaneous demixing. The composition of the interface is located on the binodal and the composition of the solution close to the support is located at the polymer-solvent axis. The composition of the bath side of the interface is located at the dilute end of the indicated tie lines.

in the polymer solution gradually shift to higher nonsolvent concentrations till finally the demixing gap is entered.

The delay time represents one of the most important parameters in the theory on immersion precipitation. The equations of Tsay and McHugh allow one to calculate the composition paths for longer time periods [111]. In Fig. 10A the polymer concentration of the polymer solution as a function of position in the film is presented. Fig. 10B demonstrates that at longer immersion times the composition of the solutions moves towards the binodal, and ultimately crosses the binodal. The composition paths in the ternary phase diagram only show the composition range present in the solution but do not tell anything
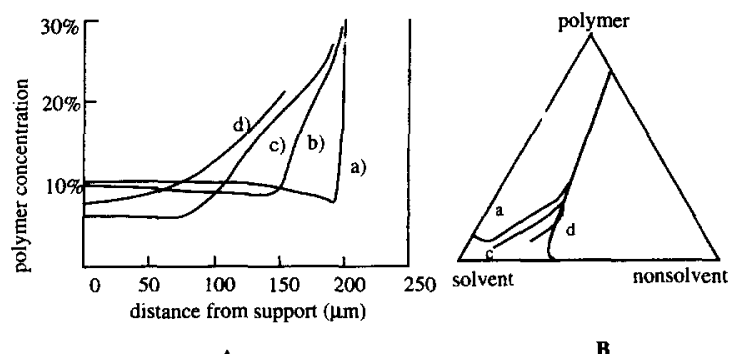

A

B

Fig. 10. Change in composition as a function of time during delayed precipitation. Delay time for the presented system: $24 \mathrm{~s}$. $a-d$ : composition paths at various times after immersion: $a=0.24$ $\mathrm{s}, \mathrm{b}=2 \mathrm{~s}, \mathrm{c}=10 \mathrm{~s}, \mathrm{~d}=24 \mathrm{~s}$. Figures adapted from [111]. (A) Polymer concentration in the solution as a function of space coordinates and time. (B) Composition paths as a function of time. 
about the location of a composition in space coordinates.

From Fig. 10 it becomes clear that the polymer concentration increases at the interface immediately after immersion. At longer time scales, the polymer concentration at the interface decreases, but the polymer concentration below the interface increases. Over time the film shrinks, because more solvent flows out of the solution than nonsolvent flows into the solution.

For an instantaneously demixing system, path II in Fig. 9, the composition path crosses the binodal immediately (delay time is zero). The polymer concentration profile at the onset of demixing is relatively flat and can be compared with profile (a) in Fig. 10B. From the discussion it becomes clear that the delay time is of crucial importance for the composition profile in the solution at the onset of demixing. The polymer concentration profile in the solution at the onset of demixing is especially important, since it will determine to a large extent the gradient in pore size over the membrane thickness and the porosity of the membrane.

As for TIPS, the morphology will be further influenced by coarsening processes and other phase transitions. These phenomena have not yet been studied for immersion precipitation and remain a challenge for future research. We have attempted to summarize the information in the literature on the influence of the input parameters in the mass transfer models on the delay time and the polymer concentration at the interface. The results are qualitatively generalized in Table 1. It should, however, be kept in mind that the influence of one specific parameter can depend on the values for other parameters. In addition, changes in one parameter can have consequences for the values of the other parameters. It has been demonstrated that the solvent-nonsolvent interaction parameter and the solvent/nonsolvent friction parameter determine to a large extent the delay time. Small changes in these parameters have an enormous impact on the predicted composition paths. Considering the uncertainty in the experimental determination of these parameters, quantitative agreement between experiment and theory cannot be expected.

Another approach was followed by Termonia [151]. A Monte Carlo diffusion model was developed to study the diffusion processes and demixing processes directly after immersion. From this model it becomes apparent that the solvent-nonsolvent interaction, here expressed as the pair interaction energy, is the major controlling factor during the diffusion. A good agreement was obtained between the calculated coagulation rate and the final membrane structure.

Furthermore, it should be kept in mind that for each application or extension of the model the assumptions have to be justified. The models have been extended to include an evaporation step $[117,118]$ or a second polymer in the polymer solution [119]. Further mass transfer models have been

Table 1

Influence of the various parameters present in the mass transfer equations on the delay time ${ }^{\text {a }}$

\begin{tabular}{lllllll}
\hline Parameter & Thickness $^{\mathrm{b}} \uparrow$ & $\chi_{12} \uparrow$ & $\chi_{23} \uparrow$ & $\chi_{13} \uparrow$ & $r\left(V_{1} / V_{3}\right) \uparrow$ & $s\left(V_{1} / V_{2}\right) \uparrow$ \\
\hline Delay time & $\uparrow$ & $\uparrow$ & - & $\downarrow$ & - & - \\
Polymer concentration ${ }^{\mathrm{c}}$ & - & $\downarrow$ & $\uparrow$ & $\uparrow$ & - \\
\hline Parameter & $\phi_{1} \uparrow^{d}$ & $\phi_{3} \uparrow^{d}$ & $\phi_{2} \uparrow^{\mathrm{e}}$ & $\zeta_{12} \uparrow / D_{12} \downarrow$ & $\zeta_{23} \uparrow / s_{23} \downarrow$ & $\zeta_{13} \uparrow / s_{13} \downarrow$ \\
\hline Delay time & $\downarrow$ & $\uparrow$ & $\uparrow^{\mathrm{f}}$ & $\uparrow$ & $\downarrow$ & $\uparrow$ \\
Polymer concentration & $-/ \downarrow$ & $\uparrow$ & $\downarrow$ & $?(-/ \downarrow)$ & $\uparrow$ & $?(-/ \downarrow)$ \\
\hline
\end{tabular}

\footnotetext{
${ }^{\text {a }}$ Increase in parameter $\uparrow$, decrease in parameter $\downarrow ;-$ no or negligible influence; ?: uncertain. $\phi_{i}$, Volume fraction component $i$. $V_{i}$, Molar volume component i. $\zeta_{\mathrm{ij}}$, Friction coefficient between component $\mathrm{j}$ and component $\mathrm{i}$. $s_{\mathrm{ij}}$, Sedimentation coefficient of component $\mathrm{j}$ in component i. $D_{\mathrm{ij}}$, Binary diffusion coefficient between component $\mathrm{i}$ and $\mathrm{j} . \mathrm{i}=1$, Nonsolvent; $\mathrm{i}=2$, solvent; $\mathrm{i}=3$ polymer.

${ }^{b}$ Initial film thickness.

${ }^{c}$ Polymer concentration of the film side of the interface at the onset of demixing.

d In solution.

e In coagulation bath.

f Sometimes $\downarrow[96,97]$.
} 
modified to include a gel layer close to the interface [120]. In addition attempts have been made to include the effect of crystallization [103].

\subsubsection{Mass transfer: experimental}

There are hardly any measurements of in situ mass transfer and visualization of phase separation processes reported in the literature. The most common procedure is to measure the light transmission through the polymer solution after immersion. Using this method the very important delay time between immersion and demixing can be quantified. Results obtained by Reuvers and Radovanovic confirmed some of the trends in delay time predicted by the mass transfer models as given in Table $1[96,97,108]$.

The growth of the phase separated region can be investigated with optical microscopy [4,78,121-125]. A droplet of polymer solution is placed between two microscope slides. Nonsolvent is subsequently introduced at the edges of the slides. Due to capillary forces, the nonsolvent is sucked between the slides. The motion of macrovoid growth and gel front can be followed as a function of time using an optical microscope. The influence of the polymer concentration on the growth rate of the precipitation front was investigated by Koenhen [78]. The growth rate decreased with increasing polymer concentration. The growth rates of both macrovoids and gel front were found to be a function of the square root of time. No details of the phase separation processes could be detected. By using much thinner capillaries van de Witte et al. were able to assess the importance of crystallization and liquid-liquid demixing for semicrystalline polymers [139].

In order to study transport of solvent into the coagulation bath, the "casting leaching method" was used $[96,122-124,126]$. For this technique, samples are periodically drawn from the coagulation bath and analysed for composition. Combined with data on the mass change of the polymer film the average composition of the film can be estimated. Using this method, one can obtain an indication of the ratio solvent outflow/nonsolvent inflow. Frommer distinguished experimentally, for the first time, the existence of the two exchange regimes (rapid demixing and delayed demixing), and related this phenomenon to the properties of the solvent/nonsolvent mixture $[122,123]$. This method was used by
Radovanovic to fit theoretical fluxes to experimental fluxes. In this way unknown transport parameters could be estimated [96]. However, the real value of this technique lies in the possibility of checking the predictions of completely independent mass transfer calculations.

Another approach was used by Frommer et al. [127]. They measured the solvent and nonsolvent flux through the polymer membrane in a diffusion cell.

Ambrosone et al. measured the full set of diffusion coefficients for a narrow composition range ( $1 \%$ PVDF, 1\% water, 98\% DMF) using Gouy interferometry [115]. They suggested that, because of the high values for the cross term diffusion coefficients involved in the solvent-nonsolvent exchange, the polymer diffuses against the concentration gradient and concentrates at the interface. The conventional explanation for the formation of the top layer is that more solvent flows out of the solution than nonsolvent flows into the solution. Both explanations are hidden in the mass transfer models, which also predict a strong increase of the polymer concentration at the interface.

More advanced experimental techniques have recently been developed. Laser techniques and spectroscopic techniques like FTIR-ATR and NMR have been used to study the composition profiles in the polymer solution $[7,114,120,128]$. The potential of spectroscopic techniques appears to be large because of the ability to identify all individual components. However, the possibilities of these techniques have not been fully explored yet. Laser techniques have been developed by McHugh and co-workers $[114,120]$. They used the refraction of laser light by refractive index gradients in solution for the monitoring of the diffusion front and reflected light for the detection of the motion of the precipitation front. In some cases a vertical optical train could be used to study the phase separation processes close to the interface.

For the film side, it has been demonstrated that the diffusion front and the gel front propagate as the square root of time up to $60 \%$ of the film thickness. The fact that the motion of the diffusion front propagates as a function of a square root of time is encouraging, since this dependence is also predicted by the transport models. The front motions accelerate 
close to the bottom of the cell. An important result is that transport processes at the film side were diffusion controlled and could be nondimensionalized. The growth rate of both the diffusion front and the gel front decreased with increasing polymer concentration in the casting solution and with increasing solvent concentrations in the coagulation bath. These observations confirmed the predictions of the model. It can be expected that much more knowledge will be gained from these set-ups in the future.

A slightly modified set-up was used to monitor the diffusion at the bath side [114]. In this case the solvent gradient at the bathside close to the interface was studied. The gradient was very high directly after immersion, and decreased rapidly in time. Increasing solvent concentrations in the coagulation bath, decreasing polymer concentrations in the solution, and increasing nonsolvent concentrations in the solution decreased the solvent gradient. Analysis of the data suggested that the transport processes at the bath side were convection controlled rather than diffusion controlled.

\subsection{Correlation of theory with membrane morphol- ogy}

\subsubsection{Introduction}

A detailed description of membrane applications and the membrane morphology that is desired for each application is presented in many books (e.g. the book written by Mulder [1]). No detailed discussion on these topics will, therefore, be presented here. It should be kept in mind that many extra process variables are available from the casting or spinning process to manipulate the morphology. In addition, the morphology and performance of the membrane can be further influenced by the introduction of extra components to the coagulation bath or the casting solution, heat treatments, evaporation steps, vapour treatment steps, multiple coagulation baths and extra coatings. More details on these topics can be found in the patent literature. In this review, only the basic parameters as mentioned in Table 1 will be briefly discussed.

There is a general consensus among researchers that, in the case of immersion precipitation, liquidliquid demixing processes are largely responsible for the membrane morphology. Nevertheless, the attribu- tion of specific structures in the membrane to phase separation processes is even more complicated for ternary systems than for binary systems. The reason for these difficulties is that the diffusion processes change the initial composition of the film directly after immersion. Also the composition is not the same throughout the solution. The mechanism of formation of the top layer can differ from the mechanism of formation for the layer close to the support. In addition, the lack of knowledge on coarsening processes complicate the analysis.

Because of the much more complex relation between membrane morphology and system parameters for the immersion precipitation process, these relations will be examined in somewhat more detail. A part of the discussion remains useful for the TIPS process. The first part of the discussion is valid for the phase separation of amorphous polymers stabilized by vitrification and to some extent also other solidification processes. In addition, some attention will be paid to the morphologies obtained for crystalline polymers.

Kimmerle distinguished four structural elements in the morphology of membranes obtained by immersion precipitation [129]:

1. Cellular structures.

2. Nodules.

3. Bicontinuous structures.

4. unconnected latex (filterdust, blushing).

A fifth membrane structure that is frequently observed is the macrovoid. Examples of these membrane structures are presented in Fig. 11. In some membranes, more than one structural element is found. Kamide et al. were able to cut slices of $1 \mu \mathrm{m}$ thickness from the top layer to bottom layer from regenerated cellulose membranes. From their micrographs a transition in structural elements can clearly be observed [130]. Similar observations were made by Gittens et al. [131].

These structures are the same structural elements that have been observed for liquid-liquid demixing in binary systems. This similarity has been recognized by most investigators in membrane formation mechanisms. Nevertheless, different mechanisms have sometimes been proposed for the formation of these structures. The current theories on the origin of the structures will be reviewed in the following section. 


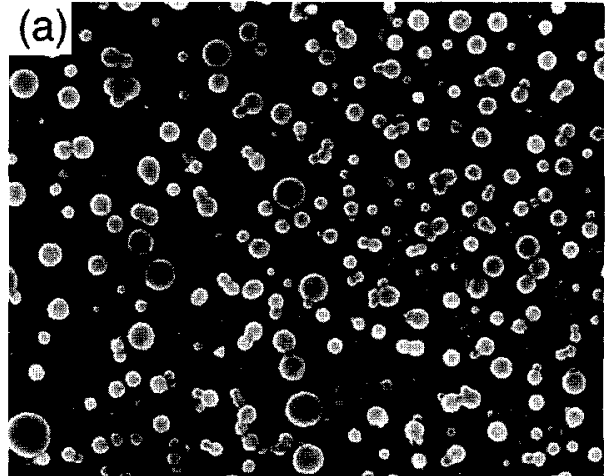
$-3^{\circ} 0^{\circ}: 60^{\circ} \cdot 0^{\circ}$ - $1000 \%$ 8. 0.00000

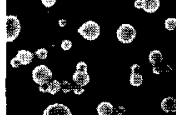

- 0 or 8 . $0_{0}^{\circ} 0^{\circ} \cdot \mathrm{C}^{\circ}=$
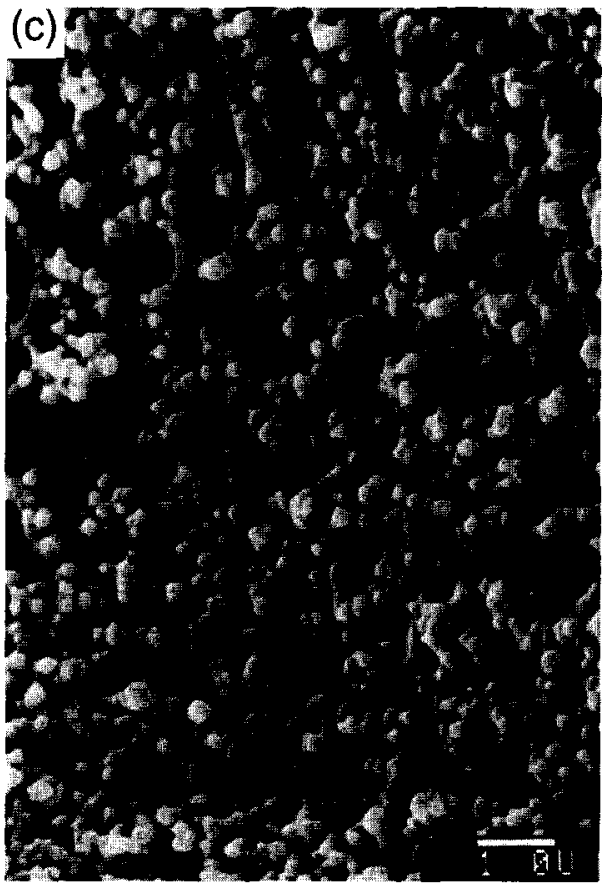

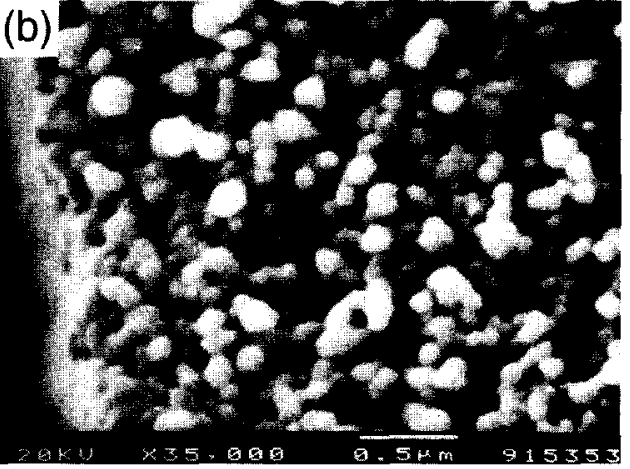

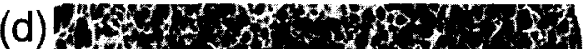
$0 \mathrm{xt}+\mathrm{t}$

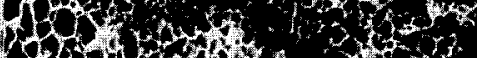

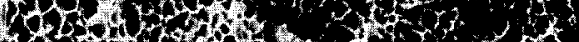

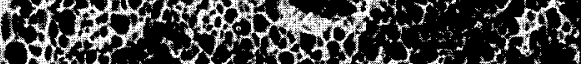

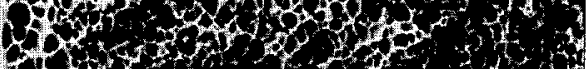

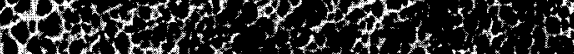

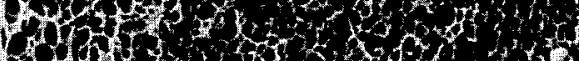

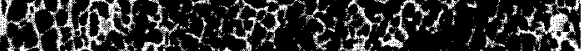

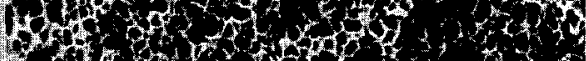

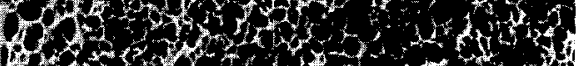

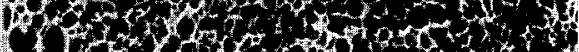

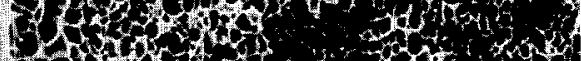

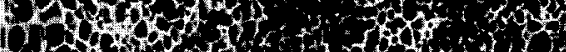

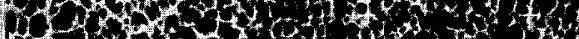

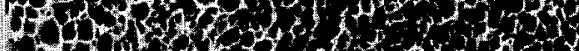

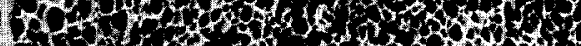

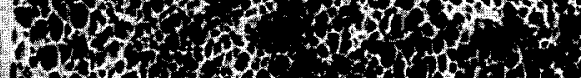

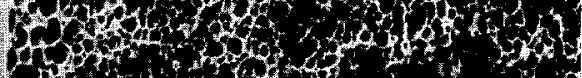
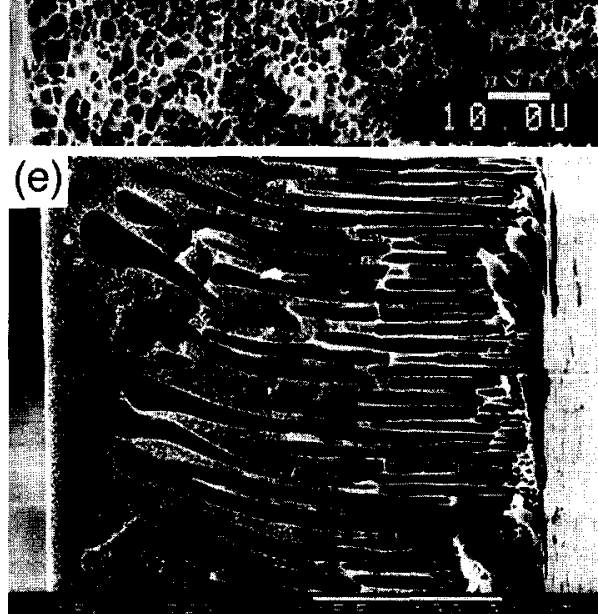


\subsubsection{Cellular structures}

Almost all membranes prepared by delayed precipitation have an open or closed cell structure in the sublayer. Smolders and co-workers demonstrated convincingly that nucleation and growth of a polymer poor phase is responsible for the pore generation $[1,124]$. In combination with the developed transport model and the phase diagrams, they were able to predict trends for the membrane structures as a function of various variables $[107,108]$.

The delay time for demixing is an important parameter in determining the membrane morphologies. Earlier in the paper the division was made between membrane morphologies obtained by rapid demixing and delayed demixing. The delay time is in the order of one second or less for rapid demixing conditions. For delayed demixing the precipitation time is in the order of seconds to minutes. Under rapid demixing conditions, membranes can be expected with a very thin top layer and a sublayer with a lot of macrovoids. The top layer of these membranes often consists of nodular structures and possesses some degree of porosity. The membrane will generally possess ultrafiltration or hyperfiltration properties, and is suitable for separation of small particles, high molecular weight components or salts from low molecular weight compounds.

For membranes obtained by delayed demixing, the top layer will be very dense and thick. Because of the high concentration of the polymer solution at the onset of demixing, the porosity and the degree of interconnectivity of the pores will be low. The permeability of the membranes is generally low. If the resistance of the sublayer can be kept low, the membranes are in principle suitable for applications based on hyperfiltration, gas separation, pervaporation and reverse osmosis. For these applications, membranes with a defect free ultrathin dense top layer and a sublayer with a high porosity and good interconnectivity of the pores are desired.

The influence of the various kinetic and thermo- dynamic parameters on the delay time is presented in Table 1 . The trends in membrane morphology can be predicted using this table and the general remarks that were mentioned earlier in this section. The influence of the composition of the casting solution and coagulation bath on the membrane morphology cannot be fully explained in terms of delay time $[97,108,82]$. The trends will be indicated in the next section.

The delay time will decrease when nonsolvent is added to the polymer solution. In addition, the size of the top layer will decrease. The pore size of the membrane also decreases with increasing polymer concentration. Close to the transition from delayed demixing to rapid demixing, macrovoids will be obtained. When the nonsolvent concentration is very high, the conditions for macrovoid formation worsen. In the model of Reuvers, the skin thickness of the nascent membrane is insufficient to sufficiently decrease the inflow of nonsolvent. Within a short period of time, demixing occurs throughout the polymer solution.

The delay time will increase when solvent is added to the coagulation bath. The polymer concentration at the interface will decrease, and facilitate phase separation in the top layer or growth of the polymer poor droplets through the interface. Wijmans demonstrated that membranes with a porous top layer can be obtained when the concentration of solvent in the coagulation bath exceeds a certain minimum value [132]. This minimum concentration is determined by the nonsolvent power. In addition, macrovoids can disappear, and thinner top layers can be obtained. Fibres with porous inner top layers are easily obtained by adding solvent to the bore liquid. The fixation of the membrane structure will also be delayed, because of the depression of the glass transition of the polymer by the solvent. Coarsening can continue for a longer time period, and the pores will become larger.

Increasing polymer concentrations will increase

Fig. 11. Micrographs of elemental membrane structures: (a) latex (produced from a 7\% solution of polyethersulfone in NMP, nonsolvent: water); (b) nodules; (c) bicontinuous structures (membrane produced from a 5\% solution of a racemic mixture of poly-L-lactide and poly-D-lactide in NMP, nonsolvent: water); (d) cellular structures (membrane produced from a 7\% solution of poly-L-lactide in chloroform, nonsolvent: methanol); (e) macrovoids (membrane produced from a 15\% solution of polyethersulfone in DMSO, nonsolvent: water/DMSO$80 / 20$ ). 
the thickness of the top layer and decrease the porosity of the membrane and the interconnectivity of the pores. The macrovoid formation will be diminished, and the pore size will increase. The polymer concentration of the casting solution also plays a role in determining the importance of other phase transitions (see later section).

\subsubsection{Nodules}

Nodules are partly fused spherical beads with a diameter of approximately $25-200 \mathrm{~nm}$, and are frequently observed in the dense top layer (the separating layer) of ultrafiltration membranes [119,133$135,141]$. The origin of these nodules is disputed in studies on membrane formation. Several theories have been developed.

One group assumes that the nodules are the result of aggregates or micelles that are already prifesent in the casting solution [135-138]. Kesting suggests that all structural elements of the membrane are built up from these aggregates. Liquid-liquid demixing by nucleation and growth of a polymer poor phase induces the formation of a porous morphology [137]. The morphological pictures of Panar [135] and the light scattering results of Kunst [95] form the basis of the theory on inhomogeneous solutions. Panar demonstrated that spherical entities could already be present in the (quenched and freeze dried) casting solution. Kunst also demonstrated that concentrated cellulose acetate solutions were to some extent ordered (hydrogen bonding network). However, Reuvers extracted gels (formed outside the demixing gap) that were obtained from concentrated polymer solutions of cellulose acetate [86]. A finely porous morphology was obtained, but no spheres were present. In addition, nodules can also be obtained from moderately concentrated polymer solutions in good solvents. The extent of ordering in these solutions can be expected to be very low. The micelle theory seems unlikely to be true in its present form, and offers no good explanation for the formation of nodules.

Another theory for the generation of nodules is that the nodules are the result of liquid-liquid demixing by nucleation and growth of a polymer rich phase. The demixing is followed by aggregation of these particles to so-called secondary particles. This theory was given a thermodynamically consis- tent basis by Kamide $[130,139,140]$. The main criticism of the theory in its present form is that no attention is paid to the transport processes before the precipitation of the polymer solution. Furthermore, solutions with low polymer concentration, and multicomponent casting solutions and coagulation baths were used for the preparation of the membranes. For nucleation and growth of a polymer rich phase, the polymer concentration of the solution has to enter the demixing gap below the critical point. The critical point for polydisperse polymers in solvent mixtures cannot easily be calculated. However, the polymer concentration of the critical point for ternary solutions and binary solutions are of comparable magnitude.

For polydisperse polymer solutions in single solvents, the polymer concentration at the critical point $\left(\phi_{p}\right)$ is given by the equation $[9,77]$ :

$\phi_{p}=\left(\left(\frac{m_{\mathrm{w}}^{2}}{m_{\mathrm{z}}}\right)^{0.5}+1\right)^{-1}$.

In this equation, $m_{\mathrm{w}}$ and $m_{\mathrm{z}}$ represent the weightand z-average degrees of polymerization (relative to the size of the solvent molecule). For membrane preparation, usually industrial polymers with relatively low molecular weights and broad distributions are used. If $m_{\mathrm{z}} / m_{\mathrm{w}}$ is 3 and $m_{\mathrm{w}}=100, \phi_{\mathrm{p}}$ amounts to $15 \%$. If solvent is linearly exchanged for nonsolvent, this also represents the maximal polymer concentration of the casting solution for nucleation and growth of a polymer rich phase.

Frequently, casting solutions contain polymer concentrations that are much higher than this value (up to $40 \%$ ). Phase separation by nucleation and growth of a polymer rich phase cannot be expected to occur in these solutions. It is necessary for the concentration of the polymer in the solution to decrease after immersion. Frommer et al. demonstrated that during the formation of membranes formed from solvent/nonsolvent combinations with high affinity, the rate of nonsolvent inflow can be higher than the rate of solvent outflow $[122,123,127]$. However, the mass transport models of Reuvers and Tsay usually predict higher polymer concentrations at the interface [107,111]. In contrast, the mass transport model of Cheng predicts that, under specific conditions, immediately after immersion rapid nonsolvent inflow 
can occur [112]. Cross term diffusion coefficients may give rise to low polymer concentrations at the interface.

Wienk et al. demonstrated that, during immersion precipitation of $10 \%$ solutions of poly(ether sulfone) in NMP in the coagulant water, most of the polymer was dispersed in the coagulation bath, indicating that in this case phase separation occurred by nucleation and growth of a polymer rich phase (see micrograph 11b) [14]. However, this theory is not necessarily suitable to explain nodule formation in concentrated polymer solutions. It also remains unclear whether the time between phase separation and solidification is sufficient to allow for nucleation and growth of a polymer rich phase.

In order to explain formation of nodules from concentrated polymer solutions, Smolders and coworkers assume that spinodal decomposition of the solutions is responsible for the nodules [134]. They state that, for rapid exchange processes, the relaxation time of the polymer is too slow to obtain a rapid thermodynamic equilibrium at the interface. It is possible that the spinodal regime can be reached. In a later publication, the fixation of nodular structures during spinodal decomposition was attributed to vitrification of the polymer rich part of the concentration fluctuations in solutions with compositions located in the spinodal [141]. The spherical morphology was due to the clustering of highly entangled polymer molecules during spinodal decomposition. However, for TIPS of polymer blends and polymer solutions, usually bicontinuous structures are obtained even in the early stage of spinodal decomposition.

It should be noted that in quenched films (from the melt or by solvent evaporation) of amorphous polymers, like polycarbonate, often nodular structures of the same size have been found [142]. These nodules are also observed in amorphous poly(ethylene terephthalate) and linear polyethylene. The nodules are paracrystalline and gradually evolve into lamellae.

It appears that no experimental evidence is available to support the current theories for nodule formation. To gain more insight into the mechanisms for nodule formation, membranes should be prepared using well characterized and completely amorphous polymers. For instance, the influence of the molecu- lar weight and polymer concentration of the casting solution on the membrane morphology would be very elucidating.

\subsubsection{Bicontinuous morphologies}

Membranes that consist, as a whole, of a highly interconnected pore structure, or contain a layer with a highly interconnected pore structure, were prepared [130,131]. These interconnected pore structures can be due to spinodal decomposition, or can be the result of coalescence of polymer poor droplets generated by binodal decomposition. Kesting and Kamide suggested that these bicontinuous structures can be due to the aggregation of nodules [136-140].

It has been argued that spinodal demixing during membrane formation is not very likely. Two reasons for this have been given. First of all, according to the mass transport model theories, the driving force for diffusion becomes zero at the spinodal $[96,97,116,150]$. Secondly, Wijmans and Smolders state that binodal demixing is usually sufficiently rapid to prevent the composition of the solution from reaching the spinodal [6].

Binder states that the binodal decomposition process and the spinodal decomposition will resemble each other in the vicinity of the spinodal [10]. It is possible that open structures can also be obtained by deep quenches in the metastable area. Still, if the cellular structures can be attributed to the nucleation and growth of polymer poor phase and the nodules to nucleation and growth of polymer rich phase, it seems reasonable to attrute the intermediate bicontinuous layer to spinodal decomposition. An additional argument for the gradual change in decomposition mechanism is that the bicontinuous structures often contain spheres (see Fig. 11c) [130,133,137].

Nevertheless, these discussions remain hypothetical and more experiments have to be performed in order to resolve this issue.

\subsection{5. macrovoids}

Macrovoids are very large elongated pores which can extend over the entire membrane thickness. Macrovoids are, in general, undesirable, because they cause mechanical weaknesses in the membrane. A lot of controversy exists concerning the origin of the 
macrovoids. Before discussing the existing theories, a number of experimental observations on macrovoid formation will be presented $[4,5,7,8,122,123,125$, $127,134,143]$.

- Microscopy studies have demonstrated that, in general, the macrovoid grows faster than the gelation front.

- Macrovoids are usually associated with instantaneous precipitation. Typical macrovoid inducing solvent/nonsolvent combinations are $\mathrm{NMP} /$ water and DMSO/water.

- Most of the techniques that can be used to delay the onset of demixing will usually also result in the disappearance of macrovoids. For example, addition of solvent to the coagulation bath will decrease the tendency for macrovoid formation.

- Increases in the viscosity of the polymer solution will decrease the tendency for macrovoid formation.

- The walls of macrovoids are usually porous. This indicates that coalescence of the macrovoids with the surrounding polymer poor droplets remains possible.

- If only a few macrovoids are present in the membrane morphology, the macrovoids have a pear shaped morphology. If many macrovoids are present, the macrovoids have a highly elongated shape.

Theories were formulated for the origin of macrovoids, starting with the early fundamental studies on membrane formation. These early theories have been recently reviewed by Smolders et al. in [134], and will not be discussed here. The other theories that are used most often to explain the formation of macrovoids will be briefly summarized.

Strathmann explained that macrovoids are the result of the rapid penetration of nonsolvent at certain weak spots in the top layer of the membrane (viscous fingering) [4,5]. There are two major objections to this theory. Firstly, large defects are almost never observed at the surface of the membrane. Secondly, this theory assumes that the walls of the macrovoids will be similar to the dense top surface of the membrane. However, macrovoids are frequently highly porous. Other theories focus on convective cells (Marangoni effect). As Frommer pointed out, most conditions required for the formation of convective cells occur in the rapid precipitation processes. One example of this is the occurrence of large viscosity differences between the two phases and steep concentration gradients. Density gradients are not responsible for the convection, and therefore it was suggested that interfacial tension differences could be responsible. In addition, large heat effects are involved in the mixing of the solvent/nonsolvent combinations typical for macrovoids (NMP/water, $\mathrm{DMSO}$ /water, DMF/water). Temperature differences can give rise to additional convection. The theory does not offer an explanation for the macrovoids that are sometimes observed in dry-cast membranes, nor does it explain how macrovoids could form at a large distance from the top surface [7].

Krantz et al. suggested that thin film instabilities could be responsible for the initiation of macrovoids $[143,144]$. They suggested that, because of the steep concentration gradients at the interface, small (random) perturbations result in a decrease of the chemical potential and evolve into periodic structures. Also this theory does not account for macrovoids which form initially at larger distances from the surface. It should be noted that, because the analysis of cross sections is only two-dimensional, it is possible that the initial formation point of the macrovoids is not present in the examined cross section.

A very elegant, but also unproven, mechanism for macrovoid formation was proposed by Smolders and Reuvers [134]. This theory focuses on the relative kinetics of the growth of polymer poor droplets and the exchange rate of solvent for nonsolvent. Due to the large amount of solvent present in the polymer poor droplets, the droplet can be viewed as a coagulation bath with a lot of solvent. Delayed demixing occurs, and a net solvent inflow from the surroundings into the droplet is the result. In order for this to occur the growth rate of the droplet must be sufficiently rapid to prevent it being taken over by the rapid precipitation front.

The very recent Monte Carlo diffusion model by Termonia again shows that the solvent-nonsolvent interaction parameter is the major controlling parameter in the solvent-nonsolvent exchange process [151]. He proposed that nonsolvent penetration through skin defects initiate the macrovoids. The faster exchange of solvent for nonsolvent through the defects was thought to be responsible for the growth stage. 


\subsubsection{Morphology of semicrystalline polymers}

The role of solid-liquid demixing in membrane formation by immersion precipitation on membrane morphology has been discussed in detail: by Van de Witte et al. for poly-L-lactide, by Bulte et al. for nylon-4,6, and by Cheng et al. for other nylons [145-147,152]. For these rapidly crystallizing polymers, membranes could be obtained with structures caused by solid-liquid demixing (spherulites, lamellar stacks), or with structures caused by liquid-liquid demixing (cellular morphologies). The polymer concentration of the casting solution and the choice of the solvent/nonsolvent system determined which morphology was obtained. Increasing polymer concentrations favoured solid-liquid demixing over liquid-liquid demixing. Indications for the influence of crystallization can also be obtained from the SEM pictures of polyvinylidene fluoride membranes and nylon-6 membranes [148,149].

Often crystallization processes are too slow to play any role in rapid membrane formation processes, and therefore membranes are obtained that are not crystalline at all (polyphenyleneoxide [98]). From the morphological pictures of Broens [124], it can be observed that under some circumstances polyphenyleneoxide in solutions of trichloroethylene/octanol precipitated in octanol can form morphologies which are the result of solid-liquid demixing. Cellulose acetate is usually only crystalline after annealing [136]. Recently evidence was presented for the presence of liquid crystalline phases in the top layer of cellulose acetate membranes [155].

\section{Conclusions}

New experimental and theoretical physical research related to the formation of morphology of polymeric membranes by phase separation of polymer solutions has been reviewed. Two typical phase separation processes have been discussed in more detail: thermally induced phase separation (TIPS) and immersion precipitation.

The processes involved in the preparation of membranes are complex. A wide variety of phase transitions (or combinations of transitions) can induce the formation of stable structures in solution. At present, many of the physical processes involved in the formation of membranes by phase separation of polymer solutions, are well understood. In spite of this, detailed prediction of membrane morphology is not easy: extensive knowledge of phase diagrams, kinetics of phase transitions, and coarsening phenomena, all in relation to resulting membrane morphologies, is required for a detailed understanding of membrane forming systems, and thus for providing reliable guidelines for preparation and optimization of membranes for specific processes.

Hence, more binary and ternary phase diagrams have to be determined in detail, in particular as a function of cooling rate and quench depth (most ternary, i.e. isothermal, phase diagrams are reconstructed from isothermal cuts through pseudo-binary temperature-composition diagrams). More data regarding solidification processes are needed: they seem to depend strongly on the polymer-solvent(nonsolvent) combination. In addition, there is a lack of quantitative knowledge regarding the kinetics of such processes. Much more viscoelastic data have to be obtained, as a function of frequency of measurement, temperature, and (above all) solution composition, in order to gain more insight into the nature and the kinetics of the many types of "gelation".

The mass transfer models have identified the process parameters that are important for the exchange processes occurring during the immersion precipitation process. However, the many assumptions (and the experimental problems in determining values for the appropriate parameters, like diffusion coefficients as a function of solution composition) involved in these models impede (even semiquantitative) predictions. It can be expected that recently developed experimental techniques for studying mass transfer and phase separation will provide valuable additional information. Still more knowledge on various aspects of membrane formation can be gained from detailed in situ microscopical studies of the phase separation of polymer solutions. The new molecular modeling methods can also provide useful insights.

Peculiar membrane structures like nodules and macrovoids most likely originate from the mass transfer. Although several plausible theories have been developed to explain the formation of these structures, hardly any direct evidence, based on experimentally checked theoretical predictions, is available to support these theories. To gain more insight, 
for instance, in the mechanisms for nodule formation, membranes should be prepared using well characterized and completely amorphous polymers. It could then be elucidating to study the influence of molecular weight and polymer concentration of the casting solution on the membrane morphology.

\section{Acknowledgements}

The authors would like to thank Dr. W. Hinrichs for his suggestions on this paper. Dr. M. Mulder is acknowledged for thoroughly reading this paper and for providing the micrographs on nodular structures. Finally, discussions with Dr. McHugh are gratefully acknowledged.

\section{References}

[1] M. Mulder, Basic Principles of Membrane Technology, Kluwer Academic, Dordrecht, The Netherlands, 1992.

[2] H.K. Lonsdale, The growth of membrane technology; J. Membrane Sci., 10 (1982) 81.

[3] W. Pusch and A. Walch, Synthetic membranes-preparation, structure and, application, Angew. Chem. Int. Ed. Engl., 21 (1982) 660.

[4] H. Strathmann, Production of microporous media by phase inversion processes, ACS Symp. Ser., 269 (1985) 165.

[5] H. Strathmann and K. Koch, The formation mechanism of phase inversion membranes, Desalination, 21 (1977) 241.

[6] J.G. Wijmans and C.A. Smolders, Preparation of asymmetric membranes by the phase inversion process, in H.K. Londsdale and M.H. Pinho (Eds.), Synthetic Membranes: Science, Engineering and Applications, Reidel, Dordrecht, The Netherlands, 1986, pp. 39-56.

[7] L. Zeman and T. Fraser, Formation of air-cast cellulose acetate membranes. Part I. Study of macrovoid formation, J. Membrane Sci., 84 (1993) 93.

[8] L. Zeman and T. Fraser, Formation of air-cast cellulose acetate membranes. Part II. Kinetics of demixing and microvoid growth, J. Membrane Sci., 87 (1994) 267.

[9] M. Kurata, Thermodynamics of Polymer Solutions, Harwood Academic, London, 1982.

[10] K. Binder, Spinodal decomposition, in P. Haasen (Ed.), Materials Science and Technology, A Comprehensive Treatment, Vol. 5, VCH, New York, 1991, pp. 405-472.

[11] J.W. Cahn, Spinodal decomposition, Trans. Metall. Soc. AIME, 242 (1968) 166.

[12] J.A. Ronner, S. Groot Wassink and C.A. Smolders, Investigation of liquid-liquid demixing and aggregate formation in a membrane forming system by means of pulse-induced critical scattering (PICS), J. Membrane Sci., 42 (1989) 27.
[13] F.-J. Tsai and J.M. Torkelson, Roles of phase separation mechanism and coarsening in the formation of poly(methyl methacrylate) asymmetric membranes, Macromolecules, 23 (1990) 775 .

[14] F.-J. Tsai and J.M. Torkelson, Microporous poly(methyl methacrylate) membranes: effect of a low-viscosity solvent on the formation mechanism, Macromolecules, 23 (1990) 4983.

[15] L.P. McMaster, Aspects of liquid-liquid phase transition phenomena in multicomponent polymeric systems, Adv. Chem. Ser., 142 (1975) 43.

[16] A. Laxminarayan, K.S. McGuire, S.S. Kim and D.R. Lloyd, Effect of initial composition, phase separation temperature and polymer crystallization on the formation of microcellular structures via thermally induced phase separation, Polymer, 35 (1994) 3060.

[17] G.T. Caneba and D.S. Soong, Polymer membrane formation through the thermal-inversion process. 2. Mathematical modelling of membrane structure formation, Macromolecules, 18 (1985) 2545.

[18] E.D. Siggia, Late stages of spinodal decomposition in binary mixtures, Phys. Rev. A, 20 (1979) 595.

[19] J.-M. Guenet, Thermoreversible Gelation of Polymers and Biopolymers, Academic Press, London, 1992.

[20] M.J. Miles, Gelation, in D.C. Bassett (Ed.), Developments in Crystalline Polymers-2, Elsevier Applied Science, New York, 1988, pp. 233-295.

[21] T. Tanaka, Phase transitions in gels, in R.S. Harland and R.K. Prud'homm (Eds.), Polyelectrolyte Gels, ACS, Washington DC, 1992, pp. 1-21.

[22] P.J. Lemstra, N.A.J.M. Van Aerle and C.W.M. Bastiaansen, Chain extended polyethylene, Polym. J., 19 (1987) 85.

[23] A. Zwijnenburg and A.J. Pennings, Longitudinal growth of polymer crystals from flowing solutions. III. Polyethylene crystals in couette flow, Colloid Polym. Sci., 254 (1976) 868.

[24] L. Mandelkern, Crystallization and melting, in C. Booth and C. Price (Eds.), Comprehensive Polymer Science, Vol. 2, Pergamon, Oxford, 1989.

[25] A.E. Woodward, Atlas of Polymer Morphology, Oxford University Press, New York, 1989.

[26] P.J. Bush, D. Pradhan and P. Ehrlich, Lamellar structure and organization in polyethylene gels crystallized from supercritical solution in propane, Macromolecules, 24 (1991) 1439.

[27] L. Mandelkern, C.O. Edwards, R.C. Domszy and M.W. Davidson, Gelation accompanying crystallization from dilute solutions: some guiding principles, in P. Dubin (Ed.), Polymer Science and Technology, Vol. 30, Plenum Press, New York/London, 1985, pp. 121-141.

[28] R.C. Domszy, R. Alamo, C.O. Edwards and L. Mandelkern, Thermoreversible gelation and crystallization of homopolymers and copolymers, Macromolecules, 19 (1986) 310.

[29] A. Prasad, H. Marand and L. Mandelkern, Supermolecular morphology of thermoreversible gels formed from homogeneous and heterogeneous solutions, J. Polym. Sci., Part B: Polym. Phys. Ed., 32 (1993) 1819. 
[30] A. Keller, in A. Hiltner (Ed.), Structure Property Relationships of Polymeric Solids, Plenum Press, New York 1983, pp. 25-57.

[31] M. Giralomo, A. Keller, K. Miyasaka and N. Overbergh, Gelation-crystallization in isotactic polystyrene solutefions and its implications to crystal morphology to the original and structure of gels and to the chemical heterogeneity of polyolefins, J. Polym. Sci., Part B: Polym. Phys. Ed., 14 (1976) 39.

[32] P.J. Flory, Principles of Polymer Chemistry, Cornell University Press, 1953.

[33] F. Debert and H. Berghmans, Phase behaviour of syndiotactic polystyrene-decalin, Polymer, 34 (1993) 2192.

[34] A. Prasad and L. Mandelkern, Thermoreversible gelation of syndiotactic polystyrene, Macromolecules, 23 (1990) 5041.

[35] T. Tanigami, H. Suzuki, K. Yamaura and S. Matsuzawa, Gelation and crystallization of poly(4-methyl pentene) in cyclohexane solution, Macromolecules, 18 (1985) 2595.

[36] J.W. Cho, H.Y. Song and S.Y. Kim, Thermoreversible gelation of poly(vinylidene fluoride) in $\gamma$-butyrolactone solution, Polymer, 34 (1993) 102.

[37] J. Spevacek and B. Schneider, Aggregation of stereoregular poly(methyl methacrylates), Adv. Colloid Interface Sci., 27 (1987) 81

[38] G.L. Loomis, J.R. Murdoch and K.H. Gardner, Polylactide stereocomplexes, Polym. Prepr., 31 (1990) 55.

[39] Y. Chatani, Y. Shimane, T. Inagaki, T. Ijitsu, T. Yukinari and H. Shikuma, Structural study on syndiotactic polystyrene: 2 . Crystal structure of molecular compound with toluene, Polymer, 34 (1993) 1620.

[40] J. Arnauts and H. Berghmans, Amorphous gels of atactic polystyrene, Polym. Commun., 28 (1987) 66.

[41] J. Arnauts, H. Berghmans and R. Koningsveld, Structure formation in solutions of atactic polystyrene in trans-decalin, Makromol. Chem., 194 (1993) 77.

[42] S. Callister, A. Keller and R.M. Hikmet, On thermoreversible gels: their classification, relation to phase transitions and vitrification, their morphology and properties, Makromol. Chem. Macromol. Symp., 39 (1990) 19.

[43] A. Labudzinska and A. Ziabicki, Effect of composition and gelation conditions on structural changes accompanying the gelation of PAN, PVA and gelatin solutions, Koll. Z. Z. Polym., 243 (1971) 21.

[44] A. Coniglio, H.E. Stanley and W. Klein, Site-bond correlated percolation problem: a statistical model of polymer gelation, Phys. Rev. Lett., 42 (1979) 518.

[45] F. Tanaka and W.H. Stockmayer, Thermoreversible gelation with junctions of variable multiplicity, Macromolecules, 27 (1994) 3943.

[46] J.E. Eldridge and J. Ferry, Studies on the cross-linking process in gelatin gels. III. Dependence of the melting point on concentration and molecular weight, J. Phys. Chem., 58 (1954) 992

[47] J.F. Joanny, Gel formation in ionomers, Polymer, 21 (1980) 71.

[48] M. Daoud and G. Jannink, Temperature concentration diagram of polymer solutions, J. Phys. (Paris), 37 (1976) 973.
[49] A. Hiltner, in S.E. Keinath, R.L. Miller and J.K. Rieke (Eds.), Order in the Amorphous State of Polymers, Plenum Press, New York, 1987, pp. 119-133.

[50] H.-M. Tan, A. Moet, A. Hiltner and E. Baer, Thermoreversible gelation of atactic polystyrene solutions, Macromolecules, 16 (1983) 28.

[51] T. Schneider, B.A. Wolf, H. Kasten and F. Kremer, Thermoreversible gelation and vitrification of highly concentrated polymer solutions under poor thermodynamic conditions, Macromolecules, 24 (1991) 5387.

[52] Y.C. Yang and P.H. Geil, Morphology and properties of PVC/solvent gels, J. Macromol. Sci., Phys. B, 22 (1983) 463.

[53] K. Kawanishi, M. Komatsu and T. Inoue, Thermodynamic considerations of the sol-gel transition in polymer solutions, Polymer, 28 (1987) 980.

[54] J.-M. Guenet and M. Klein, Structures in polystyrene solutions: their origins and their implications on physical gelation, Makromol. Chem. Macromol. Symp., 39 (1990) 85.

[55] J.M. Guenet, A. Menelle, V. Schaffhauser, P. Terech and A. Tierry, Isotactic polystyrene/cis-decaline mixtures phase diagram and molecular structures, Colloid Polym. Sci., 272 (1994) 36.

[56] H. Berghmans, P. van den Broecke and S. Thijs, Chain structure and solvent quality: key factors in the thermoreversible gelation of solutions of vinyl polymers, Integration Fundam. Polym. Sci. Technol., 4 (1989) 11-19.

[57] M. Berghmans, S. Thijs, M. Cornette, H. Berghmans, F.C. De Schryver, P. Moldenaers and J. Mewis, Thermoreversible gelation of syndiotactic poly(methyl methacrylate) in toluene: A two-step mechanism, Macromolecules, 27 (1994) 7669.

[58] B.J. Goodfellow and R.H. Wilson, A fourier transform IR study of the gelation of amylose and amylopectin, Biopolymers, 30 (1990) 1183.

[59] R.M. Hikmet, S. Callister and A. Keller, Thermoreversible gelation of atactic polystyrene: phase transformation and morphology, Polymer, 29 (1988)1378.

[60] J.H. Aubert and R.L. Clough, Low density, microcellular polystyrene foams, Polymer, 26 (1985) 2047.

[61] P. Vandeweerdt, H. Berghmans and Y. Tervoort, Temperature-concentration behavior of solutions of polydisperse, atactic poly(methylmethacrylate) and its influence on the formation of amorphous, microporous membranes, Macromolecules, 24 (1991) 3547.

[62] F.C. Frank and A. Keller, Two-fluid phase separation modified by a glass transition, Polym. Commun., 29 (1988) 186.

[63] W.R. Burghardt, Phase diagrams for binary polymer systems exhibiting both crystallization and limited liquid-liquid miscibility, Macromolecules, 22 (1989) 2482.

[64] H.K. Lee, A.S. Myerson and K. Levon, Nonequilibrium liquid-liquid phase separation in crystallizable polymer solutions, Macromolecules, 25 (1992) 4002.

[65] D.R. Lloyd, K.E. Kinzer and H.S. Tseng, Microporous membrane formation via thermally induced phase separation. I. Solid-liquid phase separation, J. Membrane Sci., 52 (1990) 239. 
[66] D.R. Lloyd, S.S. Kim and K.E. Kinzer, Microporous membrane formation via thermally induced phase separation. II. Liquid-liquid phase separation, J. Membrane Sci., 64 (1991) 1.

[67] S.S. Kim and D.R. Lloyd, Microporous membrane formation via thermally induced phase separation. III. Effect of thermodynamic interactions on the structure of isotactic propylene membranes, J. Membrane Sci., 64 (1991) 13.

[68] A.A. Alwattari and D.R. Lloyd, Microporous membrane formation via thermally induced phase separation. VI. Effect of diluent morphology and relative crystallization kinetics on polypropopylene membrane structure, J. Membrane Sci., 64 (1991) 55.

[69] G.B.A. Lim, S.S. Kim, Q. Ye, Y.F. Wang and D.R. Lloyd, Microporous membrane formation via thermally-induced phase separation. IV. Effect of isotactic polypropylene crystallization kinetics on membrane structure, J. Membrane Sci., 64 (1991) 31.

[70] J.H. Aubert, Isotactic polystyrene phase diagrams and physical gelation, Macromolecules, 21 (1988) 3468.

[71] P. Schaaf, B. Lots and J.C. Wittman, Liquid-liquid phase separation and crystallization in binary polymer systems, Polymer, 28 (1987) 193.

[72] L. Aerts, M. Kunz, H. Berghmans and R. Koningsveld, Relation between phase behaviour and morphology in polyethylene/diphenylether systems, Makromol. Chem., 194 (1993) 2697.

[73] H. Tompa, Polymer Solutions, Butterworths, London, 1956.

[74] F.W. Altena and C.A. Smolders, Calculation of liquid-liquid phase separation in a ternary system of a polymer in a mixture of a solvent and a nonsolvent, Mromolecules, 15 (1982) 1491.

[75] L. Yilmaz and A.J. McHugh, Analysis of nonsolventsolvent-polymer phase diagrams and their relevance to membrane formation modelling, J. Appl. Polym. Sci., 31 (1986) 997.

[76] K. Kamide and S. Matsuda, Phase equilibria of quasi-ternary systems consisting of multicomponents polymers in a binary solvent mixture II. Role of initial concentration and relative amount of polymers partitioned in two phases, Polym. J., 7 (1984) 515.

[77] R. Koningsveld, Liquid-liquid equilibria in quasi-ternary systems, Chem. Zvesti, 26 (1972) 263.

[78] D.M. Koenhen, M.H.V. Mulder and C.A. Smolders, Phase separation phenomena during the formation of asymmetric membranes, J. Appl. Polym. Sci., 21 (1977) 199.

[79] W.W. Lau, M.D. Guiver and T. Matsuura, Phase separation in carboxylated polysulfone/solvent/water systems, J. Appl. Polym. Sci., 42 (1991) 3215.

[80] B.T. Swinyard and J.A. Barrie, Phase separation in nonsolvent/dimethylformamide/polyethersulfone and nonsolvent/dimethylformamide/polysulfone systems, Br. Polym. J., 20 (1988) 317.

[81] Y.S. Kang, H.J. Kim and U.Y. Kim, Asymmetric membrane formation via immersion precipitation method. I. Kinetic effect, J. Membrane Sci., 60 (1991) 233.
[82] M. Mulder, J. Oude Hendrikman, J.G. Wijmans and C.A. Smolders, A rationale for the preparation of asymmetric pervaporation membranes, J. Appl. Polym. Sci., 30 (1985) 2805.

[83] L. Shuguang, J. Cheng Zhang and Z. Yuangi, The investigation of solution thermodynamics for the polysulfone DMAc water system, Desalination, 62 (1987) 79.

[84] J.G. Wijmans, J. Kant, M.H.V. Mulder, C.A. Smolders, Phase separation phenomena in solutions of polysulfone in mixtures of a solvent and a nonsolvent: relationship with membrane formation, Polymer, 26 (1985) 1539.

[85] L. Zeman and G. Tkacik, Thermodynamic analysis of a membrane forming system water/ $N$-methyl-2pyrrolidone/polyethersulfone, J. Membrane Sci., 36 (1988) 119.

[86] A.J. Reuvers, F.W. Altena and C.A. Smolders, Demixing and gelation behaviour of ternary cellulose acetate solutions, J. Polym. Sci., Part B: Polym. Phys., 24 (1986) 783.

[87] F.W. Altena, J.S. Schroder, R. Van de Huls and C.A. Smolders, Thermoreversible gelation of cellulose acetate solutions studied by differential scanning calorimetry, J. Polym. Sci., Part B: Polym. Phys. Ed., 24 (1986)1725.

[88] H. Strathmann, P. Scheible and R.W. Baker, A rationale for the preparation of Loeb-Sourirajan-type cellulose acetate membranes, J. Appl. Polym. Sci., 15 (1971) 811.

[89] M. Guillotin, C. Lemoyne, C. Noel and L. Monnerie, Physicochemical processes occurring during the formation of cellulose diacetate membranes. Research of criteria for optimizing membrane performance. IV. Cellulose diacetate-acetone-organic additive casting solutions, Desalination, 21 (1977) 165.

[90] A. Bottino, G. Camera-Roda, G. Capanelli and S. Munari, The formation of microporous polyvinylidene difluoride membranes by phase separation, J. Membrane Sci., 57 (1991) 1 .

[91] L. Utracki, Investigations of the phenomena of coacervation. Part III. Phase equilibrium in the three components system toluene-ethanol-polystyrene, J. Appl. Polym. Sci., 22 (1962) 399.

[92] M.T. So, F.R. Eirich, H. Strathmann and R.W. Baker, Preparation of asymmetric Loeb-Sourirajan membranes, Polym. Lett., 11 (1973) 201.

[93] A. Bottino, G. Capanelli, S. Munari and A. Turturro, Thermodynamic approach to prepare asymmetric membranes of polyvinylidene fluoride, in B. Sedlacek and J. Kahovec (Eds.), Synthetic Polymeric Membranes, Walter de Gruyter, 1987, pp. 141-148.

[94] C.W. Yao, R.P. Burford and A.G. Fane, Effect of coagulation conditions on structure formation and properties of membranes from aliphatic polyamides, J. Membrane Sci., 38 (1988) 113-125.

[95] B. Kunst, D. Skevin, Gj. Dezelic and J.J. Peters, A light scattering and membrane formation study on concentrated cellulose acetate solutions, J. Appl. Polym. Sci., 20 (1976) 1339.

[96] P. Radovanovic, S.W. Thiel and S.-T. Hwang, Formation of 
asymmetric polysulfone membranes by immersion precipitation. Part I. Modelling mass transport during gelation, J. Membrane Sci., 65 (1992) 213.

[97] P. Radovanovic, S.W. Thiel and S.-T. Hwang, Formation of asymmetric polysulfone membranes by immersion precipitation. Part II. The effect of casting solution and gelation bath compositions on membrane structure and skin formation, J. Membrane Sci., 65 (1992) 231.

[98] W.R. Burghardt, L. Yilmaz and A.J. McHugh, Glass transition, crystallization and thermoreversible gelation in ternary PPO solutions; relationship to asymmetric membrane formation, Polymer, 28 (1987) 2085.

[99] G.E. Gaides and A.J. McHugh, Gelation in an amorphous polymer: a discussion of its relation to membrane formation, Polymer, 30 (1989) 2118.

[100] D.M. Koenhen and C.A. Smolders, Phase separation phenomena in solutions of poly(2,6-dimethyl-1,4-phenylene oxide. II. Differential scanning calorimetry of solutions in toluene, J. Polym. Sci., 15 (1977) 167.

[101] D.M. Koenhen, A. Bakker, L. Broens, J.W.A. van den Berg and C.A. Smolders, Phase separation phenomena in solutions of poly(2,6-dimethyl-1,4-phenylene oxide). IV. Thermodynamic parameters for solutions in a series of homologous solvents: toluene to hexylbenzene, J. Polym. Sci.. Part B: Polym. Phys., 22 (1984) 2145.

[102] L.P. Cheng, A.H. Dwan and C.C. Gryte, Isothermal phase behaviour of nylon-6, -66 , and -610 polyamides in formic acid-water systems, J. Polymer Sci., Part B: Polym. Phys., 32 (1994) 1183.

[103] A. Bulte, Nylon-4,6 as Membrane Material, Ph.D. Thesis, University of Twente, The Netherlands, 1994.

[104] J.G. Wijmans and C.A. Smolders, Removal of a low molecular weight, crystallizable polymer fraction from solutions of polysulfone, Eur. Polym. J., 13 (1983) 1143.

[105] S. Makhija, K. Levon and E.M. Pearce, Gelation/crystallization of polyethersulfone, Polym. Prepr., 32 (1991) 473.

[106] C. Cohen, G.B. Tanny and S. Prager, Diffusion-controlled formation of porous structures in ternary polymer systems, J. Polym. Sci., Part B. Polym. Phys. Ed., 17 (1979) 477.

[107] A.J. Reuvers, J.W.A. van den Berg and C.A. Smolders, Formation of membranes by means of immersion precipitation. Part I. A model to describe mass transfer during immersion precipitation, J. Membrane Sci., 34 (1987) 45.

[108] A.J. Reuvers and C.A. Smolders, Formation of membranes by means of immersion precipitation. Part II. The mechanism of formation of membranes prepared from the system cellulose acetate-acetone-water, J. Membrane Sci., 34 (1987) 67.

[109] A.J. McHugh and L. Yilmaz, The diffusion equation for polymer membrane formation in ternary systems, J. Polym. Sci., Part B: Polym. Phys. Ed., 23 (1985) 1271.

[110] L. Yilmaz and A.J. McHugh, Modelling of asymmetric membrane formation. I. Critique of evaporation models and development of a diffusion equation formalism for the quench period, J. Membrane Sci., 28 (1986) 287.

[111] C.S. Tsay and A.J. McHugh, Mass transfer modeling of asymmetric membrane formation by phase inversion, $\mathrm{J}$. Polym. Sci., Part B: Polym. Phys., 28 (1990) 1327.

[112] (a) L.-P. Cheng, Y.S. Soh, A.-H. Dwan and C.C. Gryte, An improved model for mass transfer during the formation of polymeric membranes by the immersion precipitation process, J. Polym. Sci., Part B: Polym. Phys., 32 (1994) 1413; (b) L.-P. Cheng, A.-H. Dwan and C.C. Gryte, Membrane formation by isothermal precipitation in polyamide-formic acid-water systems II. Precipitation dynamics, J. Polym. Sci., Part B: Polym. Phys., 33 (1995) 223.

[113] R. Taylor and R. Krishna, Multicomponent Mass Transfer, Wiley Ser. Chem. Eng., Wiley, New York, 1993.

[114] G.E. Gaides and A.J. McHugh, Measurement and analysis of bath-side interfacial concentration gradients during phase inversion, J. Membrane Sci., 74 (1992) 83.

[115] L. Ambrosone, G. Guarino, R. Sartorio, V. Vitagliano, Diffusion in the system poly(vinylidene fluoride)-water-dimethylformamide at $20^{\circ} \mathrm{C}$. Implications for the membrane casting process, J. Membrane Sci., 45 (1989) 299.

[116] V. Vitagliano, R. Sartorio, S. Scala and D. Spaduzzi, Diffusion in a ternary system and the critical mixing point, J. Sol. Chem., 7 (1978) 605.

[117] C.S. Tsay and A.J. McHugh, Mass transfer dynamics of the evaporation step in membrane formation by phase inversion, J. Membrane Sci., 64 (1991) 81.

[118] C.S. Tsay and A.J. McHugh, The combined effects of evaporation and quench steps on asymmetric membrane formation by phase inversion, J. Polym. Sci., Part B: Polym. Phys., 29 (1991) 1261.

[119] R.M. Boom, I.M. Wienk, T. Van den Boomgaard and C.A. Smolders, Microstructures in phase inversion membranes: Part 2. The role of the polymeric additive, J. Membrane Sci., 73 (1992) 277.

[120] A.J. McHugh and C.S. Tsay, Dynamics of the phase inversion process, J. Appl. Polym. Sci., 46 (1992) 2011.

[121] H. Strathmann, K. Kock, P. Amar and R.W. Baker, The formation mechanism of asymmetric membranes, Desalination, 16 (1975) 179.

[122] M.A. Frommer and D. Lancet, The mechanism of membrane formation: membrane structures and their relation to preparation conditions, in A.K. Londsdale and H.E. Podall (Eds.), Reverse Osmosis Membrane Research, Plenum Press, New York, 1972, pp. 85-110.

[123] M. Frommer and R.M. Messalem, Mechanism of membrane formation. VI. Convective flows and large void formation during membrane precipitation, Ind. Eng. Chem. Prod. Res. Develop., 12 (1973) 328.

[124] L. Broens, D.M. Koenhen and C.A. Smolders, On the mechanism of formation of asymmetric ultra and hyperfiltration membranes, Desalination, 22 (1977) 205.

[125] R. Matz, The structure of cellulose acetate membranes. 1. The development of porous structures in anisotropic membranes, Desalination, 10 (1972) 1.

[126] F. Altena, J. Smid, J.W.A. van den Berg, J.G. Wijmans and C.A. Smolders, Diffusion of solvent from a cast CA solution, Polymer, 26 (1985) 1531. 
[127] M. Frommer, I. Feiner, O. Kedem and R. Bloch, The mechanism for formation of skinned membranes II. Equilibrium properties and osmotic flows determining membrane structure, Desalination, 7 (1970) 392.

[128] A. Viallat and S. Perez, NMR approach to properties of polymeric membranes, Kinetics of membrane formation, $\mathbf{J}$. Polym. Sci., Part: Polym. Phys., 31 (1993) 1567.

[129] K. Kimmerle and H. Strathmann, Analysis of the structuredetermining process of phase inversion membranes, Desalination, 79 (1990) 283.

[130] S.-I. Manabe, Y. Kamata, H. Iijma and K. Kamide, Some morphological characteristic of porous polymeric membranes prepared by "microphase separation method", Polym. J., 19 (1987) 391.

[131] G.J. Gittens, P.A. Hitchcock, D.C. Sammon and G.E. Wakley, The structure of cellulose acetate membranes for reverse osmosis. Part I. Membranes prepared from a dioxane based dope, Desalination, 8 (1970) 369.

[132] J.G. Wijmans, J.P.B. Baaij and C.A. Smolders, The mechanism of formation of microporous or skinned membranes produced by immersion precipitation, J. Membrane Sci., 14 (1983) 263.

[133] Y. Fujii, H. Iwatani and S. Kigoshi, Morphological structures of the membranes prepared from polymer solutions, Polym. J., 24 (1992) 737.

[134] C.A. Smolders, A.J. Reuvers, R.M. Boom and I.M. Wienk, Microstructures in phase inversion membranes. Part 1. Formation of macrovoids, J. Membrane Sci., 73 (1992) 259.

[135] M. Panar, H.H. Hoehn and R.R. Hebert, The nature of asymmetry in reverse osmosis membranes, Macromolecules, 6 (1973) 777.

[136] R.E. Kesting, Phase inversion membranes, ACS Symp. Ser., 269 (1985) 131

[137] R.E. Kesting, The four tiers of structure in integrally skinned phase inversion membranes and their relevance to the various separation regimes, J. Appl. Polym. Sci., 41 (1990) 2739.

[138] Z. Zhenxin and T. Matsuura, Discussions on the formation mechanism of surface pores in reverse osmosis, ultrafiltration, and microfiltration membranes prepared by the phase inversion process, J. Colloid Interf. Sci., 147 (1991) 307.

[139] K. Kamide, Thermodynamics of Polymer Solutions, Phase Equilibria and Critical Phenomena, Elsevier, Amsterdam, 1990.

[140] K. Kamide, H. Iijima and S. Matsuda, Thermodynamics of formation of porous polymeric membrane by phase separation method I. Nucleation and growth of nuclei, Polym. J., 25 (1993) 1113.

[141] I.M. Wienk, Th. Van den Boomgaard and C.A. Smolders, The formation of nodular structures in the top layer of ultrafiltration membranes, J. Appl. Polym. Sci., 53 (1994) 1011.

[142] P. Geil, in S.E. Keinath, R.L. Miller and J.K. Rieke (Eds.), Order in the Amorphous State of Polymers, Plenum Press, New York, 1987, pp. 83-118.

[143] R.J. Ray, W.B. Krantz and R.L. Sani, Linear stability theory model for finger formation in asymmetric membranes, J. Membrane Sci., 23 (1985) 155.

[144] F.G. Paulsen, S.S. Shojaie and W.B. Krantz, Effect of evaporation step on macrovoid formation in wet-cast polymeric membranes, J. Membrane Sci., 91 (1994) 265.

[145] A.M.W. Bulte, B. Folkers, M.H.V. Mulder and C.A. Smolders, Membranes of semicrystalline aliphatic polyamide nylon-4,6: formation by diffusion-induced phase separation, J. Appl. Polym. Sci., 50 (1993) 13.

[146] P. Van de Witte, H. Esselbrugge, A.M.P. Peters, P.J. Dijkstra, J. Feijen, R.J.J. Groenewegen, J. Smid, J. Olijslager, J.M. Schakenraad, M.J.D. Eenink and A.P. Sam, Formation of porous membranes for drug delivery systems, J. Controlled Release, 24 (1993) 61.

[147] (a) P. Van de Witte, P.J. Dijkstra, J.W.A. Van den Berg and J. Feijen, Membrane formation of polylactides, Polym. Prepr., 35 (1994) 842; (b) P, van de Witte, Polylactide Membranes. Correlation between Phase Transitions and Morphology, Ph.D. Thesis University of Twente, The Netherlands, 1994; (c) P. van de Witte, H. Esselbrugge, P.J. Dijkstra, J.W.A. van den Berg and J. Feijen, A morphological study of membranes obtained from the system polylactide-chloroform-methanol, J. Membrane Sci., in press; (d) P. van de Witte, A. Boorsma, H. Esselbrugge, P.J. Dijkstra, J.W.A. van den Berg and J. Feijen, A DSC study of phase transitions in polylactide-chloroform-methanol systems, Macromolecules, 29 (1996) 212; (e) P. van de Witte, J.W.A. van den Berg, J. Feijen, J.L. Reeve and A.J. McHugh, In situ analysis of solvent/nonsolvent exchange and phase separation processes during the membrane formation of polylactides, J. Appl. Polym. Sci., in press.

[148] H.-C. Shih, Y.-S. Yeh and H. Yasuda, Morphology of microporous poly(vinylidene fluoride) membranes studied by gas permeation and scanning electron microscopy, J. Membrane Sci., 50 (1990) 299.

[149] V.S. Soldatov, V.A. Artamonov and O.A. Mostovlyanskii, Mechanism of porous structure formation in polyamide microfiltration membranes, in B. Sedlacek and J. Kahovec (Eds.), Synthetic Polymeric Membranes, Walter de Gruyter, 1987, pp. 171-184.

[150] L.P. Cheng and C.C. Gryte, Limitation on composition change during the isothermal mass-transfer processes in systems with limited miscibility, Macromolecules, 25 (1992) 3293.

[151] Y. Termonia, Fundamentals of polymer coagulation, J. Polym. Sci., Part B: Polym. Phys., 33(1995) 279.

[152] L.-P. Cheng, A.-H. Dwan and C.C. Gryte, Membrane formation by isothermal precipitation in polyamide-formic acid-water systems I. Description of membrane morphology, J. Polym. Sci., Part B: Polym. Phys., 33 (1995) 211.

[153] K.S. McGuire, A. Laxminarayan and D.R. Lloyd, Kinetics of droplet-growth in liquid-liquid phase separation of polymer-diluent systems: experimental results, Polymer, 36 (1995) 4951.

[154] H.-F. Guo, H.-F.A. Laxminarayan, G.T. Caneba and K. Solc, Morphological studies of late-stage spinodal decom- 
position in polystyrene-cyclohexanol system, J. Appl. Polym. Sci., 55 (1995) 753.

[155] M.J. Rosa, M.N. de Pinho, M.H. Godinho and A.F. Martins, Optical polarizing studies of cellulose acetate membranes prepared by phase inversion, Mol. Cryst. Liq. Cryst., 258 (1995) 163.
[156] A.J. McHugh, C.S. Tsay, B.F. Barton and J.L. Reeve, Comments on a model for mass transfer during phase inversion, J. Polym. Sci., Part B: Polym. Phys., 33 (1995).

[157] B. Crist and A.R. Nesarikar, Coarsening in polyethylenecopolymer blends, Macromolecules, 28 (1995) 890. 